\title{
On Émery's inequality and a variation-of-constants formula
}

\author{
Markus Reiss*, Markus Riedle**, Onno van Gaans***
}

March 14, 2005

\begin{abstract}
* Weierstrass Institute for Applied Analysis and Stochastics, Mohrenstrasse 39, 10117 Berlin, Germany

** Department of Mathematics, Humboldt University Berlin, Unter den Linden 6, 10099 Berlin, Germany

*** Institute for Stochastics, Department for Mathematics and Computer Science, Friedrich Schiller University Jena, D-07740 Jena, Germany
\end{abstract}

\begin{abstract}
A generalization of Émery's inequality for stochastic integrals is shown for convolution integrals of the form $\left(\int_{0}^{t} g(t-s) Y(s-) \mathrm{d} Z(s)\right)_{t \geqslant 0}$, where $Z$ is a semimartingale, $Y$ an adapted càdlàg process, and $g$ a deterministic function. The function $g$ is assumed to be absolutely continuous with a derivative that is continuous or of bounded variation or a sum of such functions. The function $g$ may also have jumps, as long as the jump sizes are absolutely summable. The inequality is used to prove existence and uniqueness of solutions of equations of variation-of-constants type. As a consequence, it is shown that the solution of a stochastic delay differential equation with linear drift, bounded functional Lipschitz diffusion coefficient, and noise driven by a general semimartingale satisfies a variation-of-constants formula. The proof of the inequality consists of an approximation argument and a reduction to Émery's original inequality.
\end{abstract}

Key words: Émery's inequality, functional Lipschitz coefficient, linear drift, localization, semimartingale, stochastic delay differential equation, variation-ofconstants formula

2000 Mathematics Subject Classifications: 60H20, 60G48, 34K50

\section{Introduction}

In the study of long term behavior of semi-linear stochastic differential equations it is often a fruitful strategy to interpret the diffusion and the nonlinearity in the drift as perturbations of a deterministic linear equation. The equation can then be rewritten in a variation-of-constants form, from which it is usually easier to reveal information on the large time behavior (see for instance $[1,10]$ ). For stochastic delay differential equations, however, such a variation-of-constants formula seemed to be unknown. We will prove in this paper a variation-of-constants formula for stochastic delay differential equations with linear drift and a functional Lipschitz diffusion driven by a general semimartingale. The proof involves several other results that we believe are interesting as well. In particular, we present an extension of Émery's inequality for stochastic integrals.

Our approach is the following. If a solution of the variation-of-constants formula (VoC) exists, it can be shown by a Fubini argument that it also satisfies the original stochastic delay differential equation (SDDE). Since (SDDE) is known to admit a 
unique solution, we infer that this solution then satisfies (VoC). Thus it remains to prove existence of solutions of ( $\mathrm{VoC})$. The proof of existence (and uniqueness) of solutions of $(\mathrm{VoC})$ can be patterned along the standard lines as presented in [9] for stochastic differential equations. The idea there is to use localization arguments in order to reduce to a Banach fixed point argument in a suitable space of stochastic processes. The key estimate to obtain a contraction is the following inequality due to Émery, see [3] or [9, Theorem V.3]. For notations see [9, Section V.2] or Section 2 below.

Theorem 1.1 (Émery's inequality). Let $p, q, r \in[1, \infty]$ be such that $\frac{1}{p}+\frac{1}{q}=\frac{1}{r}$ (with the convention that $\left.\frac{1}{\infty}=0\right)$. Let $\left(\Omega, \mathcal{F},\left(\mathcal{F}_{t}\right)_{t}, \mathbb{P}\right)$ be a filtered probability space where the filtration $\left(\mathcal{F}_{t}\right)_{t}$ satisfies the usual conditions. For every process $(Y(t))_{t \geqslant 0}$ in $S^{p}=S^{p}[0, \infty)$ and every semimartingale $(Z(t))_{t \geqslant 0}$ in $H^{q}=H^{q}[0, \infty)$ one has that $\left(\int_{0}^{\bullet} Y(s-) \mathrm{d} Z(s)\right)_{t \geqslant 0}$ is in $H^{r}=H^{r}[0, \infty)$ and

$$
\left\|\int_{0}^{\bullet} Y(s-) \mathrm{d} Z(s)\right\|_{H^{r}} \leqslant\|Y\|_{S^{p}}\|Z\|_{H^{q}} .
$$

It turns out that for the more general equations of variation-of-constants type an extension of Émery's inequality is needed, namely for integral processes of the form $\int_{0}^{\bullet} g(\bullet-s) Y(s-) \mathrm{d} Z(s)$, where $g$ is a deterministic function. We will show the next generalization of Theorem 1.1.

Theorem 1.2. Let $1 \leqslant p \leqslant \infty, 1 \leqslant q \leqslant \infty$, and $1 \leqslant r<\infty$ be such that $\frac{1}{p}+\frac{1}{q}=\frac{1}{r}$. Let $T>0$. If $f:[0, T] \rightarrow \mathbb{R}$ is càdlàg and such that $f=g+h+j$ with $f \in C^{1}[0, T]$, $h \in B V^{1}[0, T]$, and $j \in P J B V[0, T]$, then there exists a constant $R \geqslant 0$ such that for every $Y \in S^{p}[0, T]$ and every $Z \in H^{q}[0, T]$ one has $\int_{0}^{\bullet} f(\bullet-s) Y(s-) \mathrm{d} Z(s) \in$ $H^{r}[0, T]$ and

$$
\left\|\int_{0}^{\bullet} f(\bullet-s) Y(s-) \mathrm{d} Z(s)\right\|_{H^{r}[0, T]} \leqslant R\|Y\|_{S^{p}[0, T]}\|Z\|_{H^{q}[0, T]} .
$$

Here $B V^{1}[a, b]$ denotes the space of absolutely continuous functions on the interval $[a, b]$ of which the derivative is almost everywhere equal to a function of bounded variation. The vector space $P J B V[a, b]$ consists of all $f:[a, b] \rightarrow \mathbb{R}$ such that

$$
f(t)=\sum_{j=1}^{\infty} \alpha_{j} 1_{\left[t_{j}, b\right]}(t), \quad t \in[a, b],
$$

for some $t_{j} \in[a, b]$ and $\alpha_{j} \in \mathbb{R}, j \in \mathbb{N}$, with $\sum_{j=1}^{\infty}\left|\alpha_{j}\right|<\infty$.

With the estimate of Theorem 1.2 we can prove the next result. For a definition of 'functional Lipschitz', see Definition 6.1. By $\mathbb{D}$ we denote the space of all adapted càdlàg processes on an underlying probability space $\left(\Omega, \mathcal{F},\left(\mathcal{F}_{t}\right)_{t \geqslant 0}, \mathbb{P}\right)$.

Theorem 1.3. Let $(Z(t))_{t \geqslant 0}$ be a semimartingale, let $J \in \mathbb{D}$, let $F$ be a functional Lipschitz map, and let $g:[0, \infty) \rightarrow \mathbb{R}$ be such that $\left.g\right|_{[0, T]} \in C^{1}[0, T]+B V^{1}[0, T]+$ $P J B V[0, T]$ for every $T>0$. Then the equation

$$
X(t)=J(t)+\int_{0}^{t} g(t-s) F(X)(s-) \mathrm{d} Z(s), \quad t \geqslant 0,
$$

has a unique (up to indistinguishability) solution $X \in \mathbb{D}$. If $J$ is a semimartingale, then so is $X$.

The proof of Theorem 1.2 is based on the following generalization of Émery's inequality. 
Theorem 1.4. Let $1 \leqslant p \leqslant \infty, 1 \leqslant q \leqslant \infty$, and $1 \leqslant r<\infty$ be such that $\frac{1}{p}+\frac{1}{q}=\frac{1}{r}$. Let $\left(\Omega, \mathcal{F},\left(\mathcal{F}_{t}\right)_{t}, \mathbb{P}\right)$ be a filtered probability space with the filtration $\left(\mathcal{F}_{t}\right)_{t}$ satisfying the usual conditions. Let $T>0$ and let $(Y(s, t))_{t, s \in[0, T]}$ be a process with $Y(t, \bullet) \in S^{p}=S^{p}[0, T]$ for all $t \in[0, T]$. Assume that there exist a constant $c>0$ and processes $Y^{\prime}(t, \bullet) \in S^{p}, t \in[0, T]$, such that $\sup _{s, t \in[0, T]}\left|Y^{\prime}(t, s)\right| \in L^{p}(\mathbb{P})$ and

$$
\left\|Y(\tau, \bullet)-Y(t, \bullet)-Y^{\prime}(t, \bullet)(\tau-t)\right\|_{S^{p}} \leqslant(\tau-t) c\left\|\sup _{u, s \in[t, \tau]}\left|Y^{\prime}(s, \bullet)-Y^{\prime}(u, \bullet)\right|\right\|_{S^{p}}
$$

for all $0 \leqslant t \leqslant \tau \leqslant T$. Assume also that $\sup _{s, t \in[0, T]}|Y(t, s)| \in L^{p}(\mathbb{P})$, and that

$$
\left\|\sup _{|s-u|<\delta}|Y(s, \bullet)-Y(u, \bullet)|\right\|_{S^{p}}+\left\|\sup _{|s-u|<\delta}\left|Y^{\prime}(s, \bullet)-Y^{\prime}(u, \bullet)\right|\right\|_{S^{p}} \rightarrow 0 \text { as } \delta \downarrow 0 .
$$

If $Z \in H^{q}=H^{q}[0, T]$, then $\int_{0}^{\bullet} Y(\bullet, s-) \mathrm{d} Z(s) \in H^{r}=H^{r}[0, T]$ and

$$
\left\|\int_{0}^{\bullet} Y(\bullet, s-) \mathrm{d} Z(s)\right\|_{H^{r}} \leqslant\left(\left\|\sup _{t \in[0, T]}|Y(t, \bullet)|\right\|_{S^{p}}+2\left(1+c_{r}\right) \mathcal{V}_{p}(Y)\right)\|Z\|_{H^{q}} .
$$

Here $c_{r}$ is the constant of [9, Theorem V.2] and

$$
\begin{aligned}
& \mathcal{V}_{p}(Y,[0, T])=\sup \{\left\{\sum_{j=0}^{m-1}\left\|Y\left(\tau_{j+1}, \bullet\right)-Y\left(\tau_{j}, \bullet\right)\right\|_{S^{p}}:\right. \\
&\left.0 \leqslant \tau_{0} \leqslant \tau_{1} \leqslant \cdots \leqslant \tau_{m} \leqslant T, m \in \mathbb{N}\right\} .
\end{aligned}
$$

The outline of the paper is as follows. In Section 2 we collect the preliminaries and in Section 3 we will prove Theorem 1.4 for a special class of processes $Y$ by a reduction to Theorem 1.1. Then in Section 4 we will give an approximation argument to establish Theorem 1.4. Section 5 then applies Theorem 1.4 to convolutions with deterministic functions and proves Theorem 1.2. Section 6 uses the results of Section 5 to obtain Theorem 1.3. Finally in Section 7 we use Theorem 1.3 to prove a variation-of-constants formula for stochastic delay differential equations with linear drift.

\section{Preliminaries}

\section{$2.1 \quad$ Processes}

Let us settle some notations and collect some ingredients needed for the sequel. All random variables and stochastic processes, unless stated otherwise, are assumed to be defined on a fixed filtered probability space $\left(\Omega, \mathcal{F},\left(\mathcal{F}_{t}\right)_{t}, \mathbb{P}\right)$, where the filtration $\left(\mathcal{F}_{t}\right)_{t}$ satisfies the usual conditions (see [7, Definition 1.2.25]). Let $I \subset[0, \infty)$ be an interval and let $\mathbb{D}(I)$ denote the set of all adapted processes $(X(t))_{t \in I}$ with paths that are almost surely càdlàg (that is, right continuous and the left limit exists at every $t \in I$ distinct from the left endpoint of $I)$. If $X, Y \in \mathbb{D}(I)$ satisfy $X(t)=Y(t)$ a.s. for every $t \in I$, then they are indistinguishable, that is, $X(t)=Y(t)$ for all $t \in I$ a.s. (see [7, Problem 1.1.5]). We will identify processes that are indistinguishable. Every process $X \in \mathbb{D}(I)$ is jointly measurable from $\Omega \times I \rightarrow \mathbb{R}$ (see [7, Remark 1.1.14]). For a process $X \in \mathbb{D}(I)$ and a stopping time $T$ we define the stopped process $X^{T}$ by

$$
\left(X^{T}\right)(t)(\omega)=X(t \wedge T(\omega))(\omega), \quad \omega \in \Omega, t \in I
$$


and $X^{T-}$ by

$$
\left(X^{T-}\right)(t)=\left\{\begin{array}{ll}
X(t) 1_{\{0 \leqslant t<T\}}+X(t \wedge T-) 1_{\{t \geqslant T\}} & \text { if } T(\omega)>0, \\
0 & \text { if } T(\omega)=0,
\end{array} \omega \in \Omega, t \in I .\right.
$$

Here $(X(t \wedge T-))(\omega)=\lim _{s \uparrow T(\omega)} X(t \wedge s)(\omega)$ for $\omega \in \Omega$ with $T(\omega)>0$. Stopping times are allowed to attain the value $\infty$. The jumps of a process $X \in \mathbb{D}(I)$, where $I \subset[0, \infty)$ is an interval with left endpoint $a$, are defined by $(\Delta X)(t)=X(t)-X(t-)$ for $t \in I, t \neq a$, and $(\Delta X)(a)=X(a)$. Further, by convention, $X(a-)=0$.

For an interval $I$ and a function $f: I \rightarrow \mathbb{R}$ we define the total variation of $f$ over $I$ as

$\operatorname{Var}_{I}(f)=\sup \left\{\sum_{k=0}^{m-1}\left|f\left(t_{k+1}\right)-f\left(t_{k}\right)\right|: t_{0}, \ldots, t_{m} \in I, t_{0} \leqslant t_{1} \leqslant \cdots \leqslant t_{m}, m \in \mathbb{N}\right\}$.

A process $X \in \mathbb{D}(I)$ with paths that have almost surely finite total variation over each bounded subinterval of $I$ will be called an $F V$-process and $\operatorname{Var}_{I}(X)$ is defined pathwise.

Let $L^{p}$ denote the Lebesgue space $L^{p}(\Omega, \mathcal{F}, \mathbb{P})$, where $1 \leqslant p \leqslant \infty$. For $1 \leqslant p \leqslant \infty$ and $X \in \mathbb{D}(I)$ define

$($ possibly $\infty)$ and

$$
\|X\|_{S^{p}(I)}=\left\|\sup _{t \in I}|X(t)|\right\|_{L^{p}}
$$

$$
S^{p}(I)=\left\{X \in \mathbb{D}(I):\|X\|_{S^{p}(I)}<\infty\right\} .
$$

If the interval of definition is clear from the context, we will simply write $S^{p}$ and $\|\bullet\|_{S^{p}}$.

\subsection{Semimartingales}

Let us now consider semimartingales. We adopt the definitions and notations of $[2,6]$. Recall that a process $X \in \mathbb{D}[0, \infty)$ is called a semimartingale if there exist a local martingale $M$ and an FV-process $A$ such that $X(t)=X(0)+M(t)+A(t)$ a.s. for all $t \geqslant 0$. For two semimartingales $X$ and $Y$ we denote by $[X, Y]$ their covariation (see [2, VII.42] or [6, p. 519]). For any semimartingale $X$ the process $[X, X]$ is positive and increasing (see $[6$, Theorem 26.6(ii) $]$ ). We denote $[X, X]_{\infty}=$ $\sup _{t \geqslant 0}[X, X](t)$.

We will use the above terminology also for processes $X \in \mathbb{D}[a, b]$, where $0 \leqslant$ $a \leqslant b$. We say that $X \in \mathbb{D}[a, b]$ is a local martingale (or semimartingale) if there exists a local martingale (or semimartingale) $Y \in \mathbb{D}[0, \infty)$ such that $X(t)=Y(t)$ for all $t \in[a, b]$. If $X_{1}, X_{2} \in \mathbb{D}[a, b]$ are semimartingales and $Y_{1}, Y_{2} \in \mathbb{D}[0, \infty)$ are semimartingales such that $X_{i}(t)=Y_{i}(t)$ for all $t \in[a, b], i=1,2$, then we define $\left[X_{1}, X_{2}\right](t):=\left[Y_{1}^{b}-Y_{1}^{a-}, Y_{2}^{b}-Y_{2}^{a-}\right](t), t \in[a, b]$.

For a semimartingale $Z \in \mathbb{D}[a, b]$ with $Z(a)=0$ we define

$$
\begin{gathered}
\|Z\|_{H^{p}[a, b]}=\inf \left\{\left\|[M, M](b)^{1 / 2}+\operatorname{Var}_{[a, b]}(A)\right\|_{L^{p}}: Z=M+A\right. \text { with } \\
\\
M \text { a local martingale, } A \text { an FV-process } \\
\text { and } M(a)=A(a)=0\}
\end{gathered}
$$

(possibly $\infty$ ) and let

$$
H^{p}[a, b]:=\left\{Z \text { semimartingale }: Z(a)=0,\|Z\|_{H^{p}[a, b]}<\infty\right\} .
$$

The space $H^{p}[0, \infty)$ is defined similarly by replacing the numbers in the infimum in (2.1) by $\left\|[M, M]_{\infty}^{1 / 2}+\operatorname{Var}_{[0, \infty)}(A)\right\|_{L^{p}}$. Observe that for any stopping time $T$ and any $Z \in H^{p}[a, b]$ we have $Z^{T-} \in H^{p}[a, b]$ and $\left\|Z^{T-}\right\|_{H^{p}} \leqslant\|Z\|_{H^{p}}$. 
Theorem 2.1. Let $1 \leqslant p<\infty$ and $0 \leqslant a \leqslant b$. The spaces $\left(S^{p}[a, b],\|\bullet\|_{S^{p}[a, b]}\right)$ and $\left(H^{p}[a, b],\|\bullet\|_{H^{p}[a, b]}\right)$ are Banach spaces. Moreover, if $Z \in H^{p}[a, b]$ then $Z \in S^{p}[a, b]$ and there exists a constant $c_{p}>0$ (independent of $a$ and $b$ ) such that

$$
\|Z\|_{S^{p}[a, b]} \leqslant c_{p}\|Z\|_{H^{p}[a, b]} \text { for all } Z \in H^{p}[a, b] .
$$

Proof. It is said in $\left[9\right.$, p.188-189] that $\|\bullet\|_{S^{p}[0, \infty)}$ and $\|\bullet\|_{H^{p}[0, \infty)}$ are norms. It is straightforward that $\left(S^{p}[0, \infty),\|\bullet\|_{S^{p}}[0, \infty)\right.$ is complete. Completeness of $H^{p}[0, \infty)$ endowed with $\|\bullet\|_{H^{p}[0, \infty)}$ is mentioned in [2, VII.98(e)]. The sets $\left\{X \in S^{p}[0, \infty)\right.$ : $\left.X^{b}=X, X^{a-}=0\right\}$ and $\left\{X \in H^{p}[0, \infty): X^{b}=X, X^{a}=0\right\}$ are closed subspaces of $S^{p}[0, \infty)$ and $H^{p}[0, \infty)$, respectively, and they are isometrically isomorphic to $S^{p}[a, b]$ and $H^{p}[a, b]$. The existence of $c_{p}$ is the content of [9, Theorem V.2].

The next theorem easily follows from [9, Theorem V.1, Corollary, p. 189-190].

Theorem 2.2. Let $1 \leqslant p \leqslant \infty$ and $0 \leqslant a \leqslant b$. If $Z \in H^{p}[a, b]$, then $[Z, Z](b)^{1 / 2} \in$ $L^{p}$ and

$$
\left\|[Z, Z](b)^{1 / 2}\right\|_{L^{p}} \leqslant\|Z\|_{H^{p}[a, b]} \cdot
$$

Further, if $M \in \mathbb{D}[a, b]$ is a local martingale with $M(a)=0$ and $[M, M](b)^{1 / 2} \in L^{p}$, then $M \in H^{p}[a, b]$ and

$$
\|M\|_{H^{p}[a, b]}=\left\|[M, M](b)^{1 / 2}\right\|_{L^{p}}
$$

\subsection{Stochastic integral}

We use the stochastic integral as presented in $[2,5,6]$. Let us summarize the properties that we will need.

First consider processes of the form

$$
H(t)=H_{-1} 1_{\{0\}}(t)+H_{0} 1_{\left(0, t_{1}\right]}(t)+\cdots+H_{n-1} 1_{\left(t_{n-1}, \infty\right)}(t), \quad t \geqslant 0,
$$

where $H_{-1}, H_{0}$ are $\mathcal{F}_{0}$-measurable random variables and $H_{i}$ is an $\mathcal{F}_{t_{i}}$-measurable random variable for $i \geqslant 1$ such that there exists a constant $c \in \mathbb{R}$ such that $\left|H_{i}\right|$ is bounded by $c$ for all $i$, and where $0=t_{0} \leqslant t_{1} \leqslant \cdots \leqslant t_{n}=\infty, n \in \mathbb{N}$. We denote the set of all such processes by $\mathcal{E}$. For a semimartingale $X$ and a process $H$ given by $(2.2)$ the stochastic integral is defined by

$$
(H \bullet X)(t)=\int_{0}^{t} H(s) \mathrm{d} X(s):=\sum_{i=1}^{n} H_{i-1}\left(X\left(t_{i} \wedge t\right)-X\left(t_{i-1} \wedge t\right)\right), \quad t \geqslant 0 .
$$

The predictable $\sigma$-algebra is the $\sigma$-algebra in $[0, \infty) \times \Omega$ generated by the processes $(X(t))_{t \geqslant 0}$ that are adapted to $\left(\mathcal{F}_{t-}\right)_{t \geqslant 0}$ and which have paths that are left continuous on $(0, \infty)$. Here $\mathcal{F}_{t-}$ is the $\sigma$-algebra generated by $\mathcal{F}_{s}$ with $s<t$ if $t>0$, and $F_{0-}:=\mathcal{F}_{0}$. A process $X$ is predictable if $(t, \omega) \mapsto X(t, \omega)$ is measurable with respect to the predictable $\sigma$-algebra. A process $(H(t))_{t \geqslant 0}$ is locally bounded if there exist stopping times $0=T_{0} \leqslant T_{1} \leqslant \ldots$ with $\sup _{k} T_{k}=\infty$ a.s. such that for each $k$ the random variables $H^{T_{k}}(t), t \geqslant 0$, are uniformly bounded by a constant. Observe that for any process $X \in \mathbb{D}[0, \infty)$ the process $t \mapsto X(t-)$ is both predictable and locally bounded.

The next theorem (see [2, VIII.3 and 9], [5, Theorem I.4.31 and I.4.33-37], or [6, Theorem 26.4]) extends the stochastic integral to all locally bounded predictable processes.

Theorem 2.3. Let $X$ be a semimartingale. The map $H \mapsto H \bullet X$ on $\mathcal{E}$ has a unique linear extension (also denoted by $H \mapsto H \bullet X$ ) on the space of all predictable locally 
bounded processes into the space of adapted càdlàg processes such that if $\left(H^{n}\right)_{n}$ is a sequence of predictable processes with $\left|H^{n}(t)\right| \leqslant K(t)$ for all $t \geqslant 0$ and all $n \in \mathbb{N}$ for some locally bounded predictable process $K$ and $H^{n}(t)(\omega) \rightarrow H(t)(\omega)$ for all $t \geqslant 0$ and all $\omega \in \Omega$ for some process $H$, then

$$
\left(H^{n} \bullet X\right)(t) \rightarrow(H \bullet X)(t) \text { in probability for all } t \geqslant 0 .
$$

Moreover, for every locally bounded predictable $H$ and $K$ one has

(a) $H \bullet X$ is a semimartingale;

(b) $K(H \bullet X)=(K H) \bullet X$;

(c) $\Delta(H \bullet X)$ and $H \Delta X$ are indistinguishable and $(H \bullet X)(0)=\Delta(H \bullet X)(0)=$ $H(0) X(0)$;

(d) if $X$ is a local martingale then $H \bullet X$ is a local martingale;

(e) if $X$ is of bounded variation then $H \bullet X$ is of bounded variation and

$$
\operatorname{Var}_{[0, \infty)}(H \bullet X) \leqslant \sup _{t \geqslant 0}|H(t)| \operatorname{Var}_{[0, \infty)}(X) ;
$$

(f) if $T$ is a stopping time, then $1_{[0, T]} \bullet X=X^{T}$ and $(H \bullet X)^{T}=\left(H 1_{[0, T]}\right) \bullet X=$ $H \bullet X^{T}$;

It follows in particular that the stochastic integral $\int_{0}^{t} H(s-) \mathrm{d} X(s), t \geqslant 0$, is well defined for any $H \in \mathbb{D}[0, \infty)$ and any semimartinagle $X$.

The next theorem is contained in [6, Theorem 26.6(ii) and (v)].

Theorem 2.4. If $M \in \mathbb{D}[0, \infty)$ is a local martingale and $Y$ is a locally bounded predictable process, then

$$
[Y \bullet M, Y \bullet M](t)=\int_{0}^{t} Y(s)^{2} \mathrm{~d}[M, M](s) \text { for all } t \geqslant 0 .
$$

A useful continuity property of the stochastic integral is the following $[9, \mathrm{p} .51]$.

Theorem 2.5. Let $X$ be a semimartingale. Let $\left(Y^{n}(t)\right)_{t \geqslant 0}, n \in \mathbb{N}$, be a sequence of adapted càdlàg processes and let $(Y(t))_{t \geqslant 0}$ also be an adapted càdlàg process. If

$$
\sup _{0 \leqslant s \leqslant t}\left|Y^{n}(s)-Y(s)\right| \rightarrow 0 \text { in probability for all } t \geqslant 0,
$$

then

$$
\sup _{0 \leqslant s \leqslant t}\left|\int_{0}^{s} Y^{n}(u-) \mathrm{d} X(u)-\int_{0}^{s} Y(u-) \mathrm{d} X(u)\right| \rightarrow 0 \text { in probability for all } t \geqslant 0 .
$$

We state two more results that will be needed in the next sections.

Lemma 2.6. If $f:(a, c] \rightarrow \mathbb{R}$ is right continuous at $b \in(a, c)$, then

$$
\operatorname{Var}_{(a, c]}(f)=\operatorname{Var}_{(a, b]}(f)+\operatorname{Var}_{(b, c]}(f) .
$$

Proof. From the definition of the variation it is clear that the right hand side is not more than the left hand side. Let $a<t_{0} \leqslant t_{1} \leqslant \cdots \leqslant t_{m}=c$. Then there is an $\ell \in\{1, \ldots, n-1\}$ such that $t_{\ell} \leqslant b$ and $t_{\ell+1}>b$. Therefore

$$
\begin{aligned}
& \sum_{k=0}^{m-1}\left|f\left(t_{k+1}\right)-f\left(t_{k}\right)\right| \leqslant \sum_{k=0}^{\ell-1} \mid f\left(t_{k+1}-f\left(t_{k}\right)|+| f(b)-f\left(t_{\ell}\right) \mid\right. \\
& +\left|f\left(t_{\ell+1}\right)-f(b)\right|+\sum_{k=\ell+1}^{m-1}\left|f\left(t_{k+1}\right)-f\left(t_{k}\right)\right| \\
& \leqslant \operatorname{Var}_{(a, b]}(f)+\left|f\left(t_{\ell+1}\right)-f(b)\right|+\operatorname{Var}_{(b, c]}(f) .
\end{aligned}
$$


Right continuity of $f$ at $b$ yields

$$
\operatorname{Var}_{(a, c]}(f)+\operatorname{Var}_{(a, b]}(f)+\operatorname{Var}_{(b, c]}(f) .
$$

As an example that the additivity in the above lemma does not always hold if $f$ is not right continuous, consider $f:(0,2] \rightarrow \mathbb{R}$ given by $f=1_{(1,2]}$. Then $\operatorname{Var}_{(0,1]}(f)=0, \operatorname{Var}_{(1,2]}(f)=0, \operatorname{but}_{\operatorname{Var}_{(0,2]}}(f)=1$.

The next theorem gives a description of the decomposition of the product of two semimartingales by means of stochastic integrals. Part (a) is contained in [9, Corollary 2 to Theorem II.22, p. 60] and part (b) follows from [5, Proposition I.4.49a)].

Theorem 2.7. (a) If $X$ and $Y$ in $\mathbb{D}[0, \infty)$ are semimartingales, then their product $X Y$ is a semimartingale.

(b) Let $M \in \mathbb{D}[0, \infty)$ be a local martingale. If $A \in \mathbb{D}[0, \infty)$ is an $F V$-process, then $M A$ is a semimartingale and

$$
M(t) A(t)=M(0) A(0)+\int_{(0, t]} M(s) \mathrm{d} A(s)+N(t), \quad t \geqslant 0,
$$

where $\int_{(0, \bullet]} M(s) \mathrm{d} A(s)$ is an $F V$-process and $N$ is a local martingale with $N(0)=0$ given by

$$
N(t)=\int_{(0, t]} A(s-) \mathrm{d} M(s) .
$$

\section{A special class of processes}

In this section we will show Theorem 1.4 for a special class of processes. Throughout this section, Let $T>0$, let $0=t_{0} \leqslant t_{1} \leqslant \cdots \leqslant t_{m}=T$. For $1 \leqslant k \leqslant m-1$, let $g_{k}:[0, T] \rightarrow \mathbb{R}$ be the continuous function that is 1 at $t_{k}, 0$ on $\left[0, t_{k-1}\right] \cup\left[t_{k+1}, T\right]$, and affine on $\left[t_{k-1}, t_{k}\right]$ and $\left[t_{k}, t_{k+1}\right]$. Let $g_{0}:[0, T] \rightarrow \mathbb{R}$ be the continuous function that is 1 at $t_{0}=0,0$ on $\left[t_{1}, T\right]$ and affine on $\left[0, t_{1}\right]$, and let $g_{m}:[0, T] \rightarrow \mathbb{R}$ be the continuous function that is 0 on $\left[0, t_{m-1}\right], 1$ at $T$, and affine on $\left[t_{m-1}, t_{m}\right]$.

Let $1 \leqslant p \leqslant \infty, 1 \leqslant q \leqslant \infty$, and $1 \leqslant r<\infty$ be such that $\frac{1}{p}+\frac{1}{q}=\frac{1}{r}$. Let $Y_{k} \in S^{p}[0, T], 0 \leqslant k \leqslant m-1$, and let

$$
Y(t, s)=\sum_{k=0}^{m-1} Y_{k}(s) g_{k}(t), \quad t, s \in[0, T] .
$$

Assume that $\sup _{t, s \in[0, T]}|Y(t, s)|<\infty$ a.s. Let $Z \in \mathbb{D}[0, T]$ be a semimartingale with $Z=M+A, M(0)=A(0)=0$, where $M$ is a local martingale with $[M, M](T)^{1 / 2} \in$ $L^{q}$, and $A$ an FV-process with $\operatorname{Var}_{[0, T]}(A) \in L^{q}$.

Lemma 3.1. The process $\int_{0}^{\bullet} Y(\bullet, s-) \mathrm{d} Z(s)$ is a semimartingale and

$$
\begin{aligned}
\int_{0}^{\bullet} Y(\bullet, s-) & \mathrm{d} Z(s)=\left(\int_{0}^{\bullet} \sum_{k=0}^{m-1} Y_{k}(s-) g_{k}(s-) \mathrm{d} M(s)\right) \\
+ & \left(\int_{0}^{\bullet} Y(\bullet, s-) \mathrm{d} A(s)+\sum_{k=0}^{m-1} \int_{(0, \bullet]}\left(\int_{0}^{s} Y_{k}(u-) \mathrm{d} M(u)\right) \mathrm{d} g_{k}(s)\right)
\end{aligned}
$$

where the process between the first pair of parentheses is a local martingale and the process between the second pair of parentheses an FV-process. 
Proof. We have

$$
\int_{0}^{\bullet} Y(\bullet, s-) \mathrm{d} Z(s)=\sum_{k=0}^{m-1} \int_{0}^{\bullet} Y_{k}(s-) \mathrm{d} Z(s) g_{k}(\bullet)
$$

and $g_{k}$ is a semimartingale for each $k, \int_{0}^{\bullet} Y_{k}(s-) \mathrm{d} Z(s)$ is a semimartingale for each $k$, so $\int_{0}^{\bullet} Y(\bullet, s-) \mathrm{d} Z(s)$ is a semimartingale, by Theorem 2.7 .

For each $0 \leqslant k \leqslant m-1$, Theorem 2.7 yields

$$
\begin{aligned}
\int_{0}^{t} Y_{k}(s-) \mathrm{d} M(s) g_{k}(s)=\int_{(0, t]}\left(\int_{0}^{s} Y_{k}(u-) d M(u)\right) \mathrm{d} g_{k}(t) \\
\quad+\int_{(0, t]} g_{k}(s-) \mathrm{d}\left(\int_{0}^{s} Y_{k}(u-) \mathrm{d} M(u)\right) \\
=\int_{(0, t]}\left(\int_{0}^{s} Y_{k}(u-) \mathrm{d} M(u)\right) \mathrm{d} g_{k}(s) \\
\quad+\int_{(0, t]} g_{k}(s) Y_{k}(s-) \mathrm{d} M(s), \quad t \in[0, T] .
\end{aligned}
$$

Hence

$$
\begin{aligned}
\int_{0}^{t} Y(t, s-) & d Z(s)=\int_{(0, t]} \sum_{k=0}^{m-1} g_{k}(s) Y_{k}(s-) \mathrm{d} M(s) \\
& +\int_{0}^{t} Y(t, s-) \mathrm{d} A(s)+\sum_{k=0}^{m-1} \int_{(0, t]}\left(\int_{0}^{s} Y_{k}(u-) \mathrm{d} M(u)\right) \mathrm{d} g_{k}(s),
\end{aligned}
$$

$t \in[0, T]$. A glance at Theorem 2.3(d)-(e) completes the proof.

Lemma 3.2. We have

$$
\begin{aligned}
& {\left[\int_{0}^{\bullet} \sum_{k=0}^{m-1} Y_{k}(s-) g_{k}(s-) \mathrm{d} M(s), \int_{0}^{\bullet} \sum_{k=0}^{m-1} Y_{k}(s-) g_{k}(s-) \mathrm{d} M(s)\right](T)} \\
& \quad \leqslant \sup _{t, s \in[0, T]}|Y(t, s)|^{2}[M, M](T)
\end{aligned}
$$

Proof. For $t \in[0, T]$, we have according to Theorem 2.4,

$$
\begin{aligned}
& {\left[\int_{0}^{\bullet} \sum_{k=0}^{m-1} Y_{k}(s-) g_{k}(s) \mathrm{d} M(s), \int_{0}^{\bullet} \sum_{k=0}^{m-1} Y_{k}(s-) g_{k}(s) \mathrm{d} M(s)\right](T)} \\
& \quad=\int_{0}^{t}\left|\sum_{k=0}^{m-1} Y_{k}(s-) g_{k}(s)\right|^{2} \mathrm{~d}[M, M](s) \\
& \quad \leqslant \int_{0}^{t}\left(\sup _{0 \leqslant k \leqslant m-1} \sup _{0 \leqslant u \leqslant t}\left|Y_{k}(u)\right| \sum_{k=0}^{m-1} g_{k}(s)\right)^{2} \mathrm{~d}[M, M](s) \\
& \quad \leqslant \sup _{u, s \in[0, T]}|Y(u, s)|^{2}[M, M](T) .
\end{aligned}
$$

Lemma 3.3. We have

$$
\begin{aligned}
& \operatorname{Var}_{[0, T]}\left(\int_{0}^{\bullet} Y(\bullet, s-) \mathrm{d} A(s)\right) \\
& \quad \leqslant\left(\sup _{u, s \in[0, T]}|Y(u, s)|+2 \sum_{k=0}^{m-1} \sup _{s \in[0, T]}\left|Y_{k+1}(s)-Y_{k}(s)\right|\right) \operatorname{Var}_{[0, T]}(A) .
\end{aligned}
$$


Proof. It suffices to consider partitions that are refinements of $\left(t_{k}\right)_{k}$. Let for each $k \in\{1, \ldots, m\}$,

$$
t_{k-1}=\tau_{0}^{k} \leqslant \tau_{1}^{k} \leqslant \cdots \leqslant \tau_{j_{k}}^{k}=t_{k} .
$$

Then, since $g_{k-1}=1-g_{k}$ on $\left[t_{k-1}, t_{k}\right]$,

$$
\begin{aligned}
& \sum_{k=1}^{m} \sum_{j=1}^{j_{k}}\left|\int_{0}^{\tau_{j}^{k}} Y\left(\tau_{j}^{k}, s-\right) \mathrm{d} A(s)-\int_{0}^{\tau_{j-1}^{k}} Y\left(\tau_{j-1}^{k}, s-\right) \mathrm{d} A(s)\right| \\
& =\sum_{k=1}^{m} \sum_{j=1}^{j_{k}} \mid \int_{0}^{\tau_{j}^{k}}\left(Y_{k}(s-) g_{k}\left(\tau_{j}^{k}\right)+Y_{k-1}(s-) g_{k-1}\left(\tau_{j}^{k}\right)\right) \mathrm{d} A(s) \\
& -\int_{0}^{\tau_{j-1}^{k}}\left(Y_{k}(s-) g_{k}\left(\tau_{j-1}^{k}\right)+Y_{k-1}(s-) g_{k-1}\left(\tau_{j-1}^{k}\right)\right) \mathrm{d} A(s) \\
& =\sum_{k=1}^{m} \sum_{j=1}^{j_{k}} \mid \int_{0}^{\tau_{j}^{k}}\left(Y_{k}(s-)-Y_{k-1}(s-)\right) g_{k}\left(\tau_{j}^{k}\right) \mathrm{d} A(s) \\
& +\int_{0}^{\tau_{j}^{k}} Y_{k-1}(s-) \mathrm{d} A(s) \\
& -\int_{0}^{\tau_{j-1}^{k}}\left(Y_{k}(s-)-Y_{k-1}(s-)\right) g_{k}\left(\tau_{j-1}^{k}\right) \mathrm{d} A(s) \\
& -\int_{0}^{\tau_{j-1}^{k}} Y_{k-1}(s-) \mathrm{d} A(s) \\
& =\sum_{k=1}^{m} \sum_{j=1}^{j_{k}} \mid \int_{\tau_{j-1}^{k}}^{\tau_{j}^{k}} Y_{k-1}(s-) \mathrm{d} A(s) \\
& +\int_{0}^{\tau_{j-1}^{k}}\left(Y_{k}(s-)-Y_{k-1}(s-)\right) \mathrm{d} A(s)\left(g_{k}\left(\tau_{j}^{k}\right)-g_{k}\left(\tau_{j-1}^{k}\right)\right) \\
& +\int_{\tau_{j-1}^{k}}^{\tau_{j}^{k}}\left(Y_{k}(s-)-Y_{k-1}(s-)\right) \mathrm{d} A(s) g_{k}\left(\tau_{j}^{k}\right) \\
& \leqslant \sum_{k=1}^{m} \sum_{j=1}^{j_{k}} \sup _{s \in[0, T]}\left|Y_{k-1}(s)\right| \operatorname{Var}_{\left(\tau_{j-1}^{k}, \tau_{j}^{k}\right]}(A) \\
& +\sum_{k=1}^{m} \sum_{j=1}^{j_{k}} \sup _{s \in[0, T]}\left|Y_{k}(s)-Y_{k-1}(s)\right| \operatorname{Var}_{[0, T]}(A)\left(g_{k}\left(\tau_{j}^{k}\right)-g_{k}\left(\tau_{j-1}^{k}\right)\right) \\
& +\sum_{k=1}^{m} \sum_{j=1}^{j_{k}} \sup _{s \in[0, T]}\left|Y_{k}(s)-Y_{k-1}(s)\right| \operatorname{Var}_{\left(\tau_{j-1}^{k}, \tau_{j}^{k}\right]}(A) \\
& \leqslant \sup _{k} \sup _{s \in[0, T]}\left|Y_{k-1}(s)\right| \operatorname{Var}_{[0, T]}(A)+\sum_{k=1}^{m} \sup _{s \in[0, T]}\left|Y_{k}(s)-Y_{k-1}(s)\right| \operatorname{Var}_{[0, T]}(A) \\
& +\sum_{k=1}^{m} \sup _{s \in[0, T]}\left|Y_{k}(s)-Y_{k-1}(s)\right| \operatorname{Var}_{[0, T]}(A),
\end{aligned}
$$

where for the addition of the variations of $A$ we have use Lemma 2.6. 
Lemma 3.4. We have

$$
\begin{aligned}
& \operatorname{Var}_{[0, T]}\left(\sum_{k=0}^{m-1} \int_{(0, \bullet]} \int_{0}^{s} Y_{k}(u-) \mathrm{d} M(u) \mathrm{d} g_{k}(s)\right) \\
& \leqslant 2 \sum_{k=0}^{m-1} \sup _{s \in[0, T]}\left|\int_{0}^{s}\left(Y\left(t_{k+1}, u-\right)-Y\left(t_{k}, u-\right)\right) \mathrm{d} M(u)\right|,
\end{aligned}
$$

where by convention $Y_{m}:=0$.

Proof. Set $t_{-1}=0$. For $t \in[0, T]$,

$$
\begin{aligned}
\sum_{k=0}^{m-1} \int_{(0, t]} \int_{0}^{s} Y_{k}(u-) \mathrm{d} M(u) \mathrm{d} g_{k}(s) & \int_{k=0}^{s} Y_{k}(u-) \mathrm{d} M(u) \mathrm{d} g_{k}(s) \\
= & \quad \int_{(0, t] \cap\left(t_{k-1}, t_{k}\right]} \int_{0}^{s-1}(0, t] \cap\left(t_{k}, t_{k+1}\right] \\
& \left.\int_{0}^{s} Y_{k}(u-) \mathrm{d} M(u) \mathrm{d} g_{k}(s)\right) \\
= & \sum_{k=0}^{m-1}\left(\int_{(0, t] \cap\left(t_{k-1}, t_{k}\right]} \int_{0}^{s} Y_{k}(u-) \mathrm{d} M(u) \mathrm{d} g_{k}(s)\right. \\
= & \sum_{k=1}^{s} \int_{(0, t] \cap\left(t_{k-1}, t_{k}\right]} \int_{0}^{s}\left(Y_{k}(u-) d M(u) \mathrm{d} g_{k+1}(s)\right)
\end{aligned}
$$

So

$$
\begin{aligned}
& \operatorname{Var}_{[0, T]}\left(\sum_{k=0}^{m-1} \int_{(0, \bullet]} \int_{0}^{s} Y_{k}(u-) \mathrm{d} M(u) \mathrm{d} g_{k}(s)\right) \\
& \leqslant 2 \sum_{k=1}^{m} \sup _{s \in\left(t_{k-1}, t_{k}\right]}\left|\int_{0}^{s}\left(Y_{k}(u-)-Y_{k-1}(u-)\right) \mathrm{d} M(u)\right| \\
& \quad=2 \sum_{k=1}^{m} \sup _{s \in[0, T]}\left|\int_{0}^{s}\left(Y\left(t_{k}, u-\right)-Y\left(t_{k-1}, u-\right)\right) \mathrm{d} M(u)\right| .
\end{aligned}
$$

Lemma 3.5. We have

$$
\begin{aligned}
& \left\|\int_{0}^{\bullet} Y(\bullet, s-) \mathrm{d} Z(s)\right\|_{H^{r}[0, T]} \\
& \leqslant\left(\left\|\sup _{t, s \in[0, T]}|Y(t, s)|\right\|_{L^{p}}+2\left(1+c_{r}\right) \sum_{k=0}^{m-1}\left\|\sup _{s \in[0, T]}\left|Y_{k+1}(s)-Y_{k}(s)\right|\right\|_{L^{p}}\right) \\
& \quad \cdot\left\|[M, M](T)^{1 / 2}+\operatorname{Var}_{[0, T]}(A)\right\|_{L^{q}}
\end{aligned}
$$

and in particular, $\int_{0}^{\bullet} Y(\bullet, s-) \mathrm{d} Z(s) \in H^{r}[0, T]$. 
Proof. The Lemmas 3.1-3.4 together yield

$$
\begin{aligned}
& \left\|\int_{0}^{\bullet} Y(\bullet, u-) \mathrm{d} Z(u)\right\|_{H^{r}[0, T]} \\
& \leqslant \|\left[\sum_{k=0}^{m-1} \int_{0}^{\bullet} g_{k}(s-) \mathrm{d}_{s}\left(\int_{0}^{s} Y_{k}(\sigma-) \mathrm{d} M(\sigma)\right),\right. \\
& \left.\sum_{k=0}^{m-1} \int_{0}^{\bullet} g_{k}(s-) \mathrm{d}_{s}\left(\int_{0}^{s} Y_{k}(\sigma-) \mathrm{d} M(\sigma)\right)\right]^{1 / 2} \\
& +\operatorname{Var}_{[0, T]}\left(\int_{0}^{\bullet} Y(\bullet, s-) \mathrm{d} A(s)+\sum_{k=0}^{m-1} \int_{(0, \bullet]}\left(\int_{0}^{s} Y_{k}(\sigma-) \mathrm{d} M(\sigma)\right) d g_{k}(s)\right) \|_{L^{r}} \\
& \leqslant \| \sup _{t, s \in[0, T]}|Y(t, s)|[M, M](T)^{1 / 2} \\
& +\left(\sup _{t, s \in[0, T]}|Y(t, s)|+2 \sum_{k=0}^{m-1} \sup _{s \in[0, T]}\left|Y_{k+1}(s)-Y_{k}(s)\right|\right) \operatorname{Var}_{[0, T]}(A) \\
& +2 \sum_{k=0}^{m-1} \sup _{s \in[0, T]}\left|\int_{0}^{s}\left(Y_{k+1}(\sigma-)-Y_{k}(\sigma-)\right) \mathrm{d} M(\sigma)\right|\|\|_{L^{r}} \\
& \leqslant\left\|\left(\sup _{t, s \in[0, T]}|Y(t, s)|\right)\left([M, M](T)^{1 / 2}+\operatorname{Var}_{[0, T]}(A)\right)\right\|_{L^{r}} \\
& +2\left\|\left(\sum_{k=0}^{m-1} \sup _{s \in[0, T]}\left|Y_{k+1}(s)-Y_{k}(s)\right|\right) \operatorname{Var}_{[0, T]}(A)\right\|_{L^{r}} \\
& +2\left\|\sum_{k=0}^{m-1} \sup _{s \in[0, T]} \mid \int_{0}^{s}\left(Y_{k+1}(\sigma-)-Y_{k}(\sigma-)\right) \mathrm{d} M(\sigma)\right\| \|_{L^{r}} \\
& \leqslant\left\|\sup _{t, s \in[0, T]}|Y(t, s)|\right\|_{L^{p}}\left\|[M, M](T)^{1 / 2}+\operatorname{Var}_{[0, T]}(A)\right\|_{L^{q}} \\
& +2 \sum_{k=0}^{m-1}\left\|\sup _{s \in[0, T]}\left|Y_{k+1}(s)-Y_{k}(s)\right|\right\|_{L^{p}}\left\|\operatorname{Var}_{[0, T]}(A)\right\|_{L^{q}} \\
& +2 \sum_{k=0}^{m-1}\left\|\int_{0}^{\bullet}\left(Y_{k+1}(\sigma-)-Y_{k}(\sigma-)\right) d M(\sigma)\right\|_{S^{r}[0, T]}=(*)
\end{aligned}
$$

Since by Émery's inequality and Theorem 2.2

$$
\begin{aligned}
& \left\|\int_{0}^{\bullet}\left(Y_{k+1}(\sigma-)-Y_{k}(\sigma-)\right) \mathrm{d} M(\sigma)\right\|_{S^{r}[0, T]} \\
& \quad \leqslant c_{r}\left\|\int_{0}^{\bullet}\left(Y_{k+1}(\sigma-)-Y_{k}(\sigma-)\right) \mathrm{d} M(\sigma)\right\|_{H^{r}[0, T]} \\
& \quad \leqslant c_{r}\left\|Y_{k+1}(\bullet)-Y_{k}(\bullet)\right\|_{S^{p}}\|M\|_{H^{q}[0, T]} \\
& \quad=c_{r}\left\|\sup _{s \in[0, T]} \mid Y_{k+1}(s)-Y_{k}(s)\right\|\left\|_{L^{p}}\right\|[M, M](T)^{1 / 2} \|_{L^{q}},
\end{aligned}
$$


we obtain

$$
\begin{aligned}
& (*) \leqslant\left\|\sup _{t, s \in[0, T]}|Y(t, s)|\right\|_{L^{p}}\left\|[M, M](T)^{1 / 2}+\operatorname{Var}_{[0, T]}(A)\right\|_{L^{q}} \\
& +2 \sum_{k=0}^{m-1}\left\|\sup _{s \in[0, T]} \mid Y_{k+1}(s)-Y_{k}(s)\right\|_{L^{p}}\left\|\operatorname{Var}_{[0, T]}(A)\right\|_{L^{q}} \\
& +2 c_{r} \sum_{k=0}^{m-1}\left\|\sup _{s \in[0, T]}\left|Y_{k+1}(s)-Y_{k}(s)\right|\right\|_{L^{p}}\left\|[M, M](T)^{1 / 2}\right\|_{L^{q}} \\
& \leqslant\left(\left\|\sup _{t, s \in[0, T]}|Y(t, s)|\right\|_{L^{p}}+2\left(1+c_{r}\right) \sum_{k=0}^{m-1}\left\|\sup _{s \in[0, T]}\left|Y_{k+1}(s)-Y_{k}(s)\right|\right\|_{L^{p}}\right) \\
& \text {. }\left\|[M, M](T)^{1 / 2}+\operatorname{Var}_{[0, T]}(A)\right\|_{L^{q}} .
\end{aligned}
$$

Corollary 3.6. If we assume in addition that $\mathcal{V}_{p}(H,[0, T])<\infty$, then

$$
\begin{aligned}
& \left\|\int_{0}^{\bullet} Y(\bullet, s-) \mathrm{d} Z(s)\right\|_{H^{r}[0, T]} \\
& \quad \leqslant\left(\left\|\sup _{t, s \in[0, T]}|Y(t, s)|\right\|_{L^{p}}+2\left(1+c_{r}\right) \mathcal{V}_{p}(Y,[0, T])\right)\|Z\|_{H^{q}[0, T]} .
\end{aligned}
$$

\section{Approximation argument}

Throughout this section, let $1 \leqslant p \leqslant \infty$, let $T>0$, and let $(Y(t, s))_{t, s \in[0, T]}$ be a process such that $Y(t, \bullet) \in S^{p}[0, T]$ for all $t \in[0, T]$ and such that there exists a constant $C$ and for every $t \in[0, T]$ there exists an $Y^{\prime}(t, \bullet) \in S^{p}[0, T]$ such that $\sup _{t, s \in[0, T]}\left|Y^{\prime}(t, s)\right| \in L^{p}$ and

$$
\left\|Y(\tau, \bullet)-Y(t, \bullet)-Y^{\prime}(t, \bullet)(\tau-t)\right\|_{S^{p}[0, T]} \leqslant(\tau-t) C\left\|\sup _{u, s \in[t, \tau]}\left|Y^{\prime}(s, \bullet)-Y^{\prime}(u, \bullet)\right|\right\|_{S^{p}[0, T]}
$$

for every $0 \leqslant t \leqslant \tau \leqslant T$.

Lemma 4.1. Let $0=t_{0} \leqslant t_{1} \leqslant \cdots t_{m}=T$ and let $g_{0}, \ldots, g_{m}$ be as defined in the first paragraph of Section 3. Let

$$
J(t, s):=\sum_{k=0}^{m-1} Y\left(t_{k}, s\right) g_{k}(t), \quad t, s \in[0, T] .
$$

If $\left(\tau_{\ell}^{k}\right)_{k, \ell}$ is a refinement of $\left(t_{k}\right)_{k}$ and for each $1 \leqslant k \leqslant m$,

$$
t_{k-1}=\tau_{0}^{k} \leqslant \tau_{1}^{k} \leqslant \cdots \leqslant \tau_{j_{k}}^{k}=t_{k},
$$

then

$$
\begin{gathered}
\sum_{k=1}^{m} \sum_{j=1}^{j_{k}}\left\|\left(J\left(\tau_{j}^{k}, \bullet\right)-Y\left(\tau_{j}^{k}, \bullet\right)\right)-\left(J\left(\tau_{j-1}^{k}, \bullet\right)-Y\left(\tau_{j-1}^{k}, \bullet\right)\right)\right\|_{S^{p}[0, T]} \\
\leqslant(1+2 C) T \sup _{k=1, \ldots, m}\left\|\sup _{u, s \in\left[t_{k-1}, t_{k}\right]} \mid Y^{\prime}(u, \bullet)-Y^{\prime}(s, \bullet)\right\| \|_{S^{p}[0, T]}
\end{gathered}
$$


Proof. Since $g_{k}=1-g_{k-1}$ on $\left[t_{k-1}, t_{k}\right]$, we obtain

$$
\begin{aligned}
& \sum_{k=1}^{m} \sum_{j=1}^{j_{k}}\left\|\left(J\left(\tau_{j}^{k}, \bullet\right)-Y\left(\tau_{j}^{k}, \bullet\right)\right)-\left(J\left(\tau_{j-1}^{k}, \bullet\right)-Y\left(\tau_{j-1}^{k}, \bullet\right)\right)\right\|_{S^{p}} \\
& =\sum_{k=1}^{m} \sum_{j=1}^{j_{k}} \|\left(Y\left(t_{k-1}, \bullet\right) g_{k-1}\left(\tau_{j}^{k}\right)+Y\left(t_{k}, \bullet\right) g_{k}\left(\tau_{j}^{k}\right)-Y\left(\tau_{j}^{k}, \bullet\right)\right) \\
& -\left(Y\left(t_{k-1}, \bullet\right) g_{k-1}\left(\tau_{j-1}^{k}\right)+Y\left(t_{k}, \bullet\right) g_{k}\left(\tau_{j-1}^{k}\right)-Y\left(\tau_{j-1}^{k}, \bullet\right)\right) \|_{S^{p}} \\
& =\sum_{k=1}^{m} \sum_{j=1}^{j_{k}} \| Y\left(t_{k-1}, \bullet\right)+\left(Y\left(t_{k}, \bullet\right)-Y\left(t_{k-1}, \bullet\right)\right) g_{k}\left(\tau_{j}^{k}\right)-Y\left(\tau_{j}^{k}, \bullet\right) \\
& -\left(Y\left(t_{k-1}, \bullet\right)+\left(Y\left(t_{k}, \bullet\right)-Y\left(t_{k-1}, \bullet\right)\right) g_{k}\left(\tau_{j-1}^{k}\right)-Y\left(\tau_{j-1}^{k}, \bullet\right)\right) \|_{S^{p}} \\
& \leqslant \sum_{k=1}^{m} \sum_{j=1}^{j_{k}} \|\left(Y\left(t_{k}, \bullet\right)-Y\left(t_{k-1}, \bullet\right)\right)\left(g_{k}\left(\tau_{j}^{k}\right)-g_{k}\left(\tau_{j-1}^{k}\right)\right) \\
& -\left(Y\left(\tau_{j}^{k}, \bullet\right)-Y\left(\tau_{j-1}^{k}, \bullet\right)\right) \|_{S^{p}} \\
& \leqslant \sum_{k=1}^{m} \sum_{j=1}^{j_{k}}\left(\| Y^{\prime}\left(t_{k-1}, \bullet\right)\left(t_{k}-t_{k-1}\right)\left(g_{k}\left(\tau_{j}^{k}\right)-g\left(\tau_{j-1}^{k}\right)\right)\right. \\
& -Y^{\prime}\left(\tau_{j-1}^{k}, \bullet\right)\left(\tau_{j}^{k}-\tau_{j-1}^{k}\right) \|_{S^{p}} \\
& +\left(t_{k}-t_{k-1}\right) C\left(g_{k}\left(\tau_{j}^{k}\right)-g_{k}\left(\tau_{j-1}^{k}\right)\right)\left\|\sup _{u, s \in\left[t_{k-1}, t_{k}\right]}\left|Y^{\prime}(u, \bullet)-Y^{\prime}(s, \bullet)\right|\right\|_{S^{p}} \\
& \left.+\left(\tau_{j}^{k}-\tau_{j-1}^{k}\right) C\left\|\sup _{u, s \in\left[\tau_{j-1}^{k}, \tau_{j}^{k}\right]}\left|Y^{\prime}(u, \bullet)-Y^{\prime}(s, \bullet)\right|\right\|_{S^{p}}\right) \\
& \leqslant \sum_{k=1}^{m} \sum_{j=1}^{j_{k}}\left(\left\|\left(Y^{\prime}\left(t_{k-1}, \bullet\right)-Y^{\prime}\left(\tau_{j-1}^{k}, \bullet\right)\right)\left(\tau_{j}^{k}-\tau_{j-1}^{k}\right)\right\|_{S^{p}}\right. \\
& \left.+\left(\tau_{j}^{k}-\tau_{j-1}^{k}\right) 2 C\left\|\sup _{u, s \in\left[t_{k-1}, t_{k}\right]}\left|Y^{\prime}(u, \bullet)-Y^{\prime}(s, \bullet)\right|\right\|_{S^{p}}\right) \\
& \leqslant \sum_{k=1}^{m} \sum_{j=1}^{j_{k}}(1+2 C)\left(\tau_{j}^{k}-\tau_{j-1}^{k}\right)\left\|\sup _{t_{k-1} \leqslant u, s \leqslant t_{k}}\left|Y^{\prime}(u, \bullet)-Y^{\prime}(s, \bullet)\right|\right\|_{S^{p}} \\
& \leqslant(1+2 C) t_{m} \sup _{k=1, \ldots, m}\left\|\sup _{t_{k-1} \leqslant u, s \leqslant t_{k}}\left|Y^{\prime}(u, \bullet)-Y^{\prime}(s, \bullet)\right|\right\|_{S^{p}} .
\end{aligned}
$$

Lemma 4.2. Assume that $t \mapsto Y^{\prime}(t, \bullet)$ is uniformly continuous in the sense that

$$
\left\|\sup _{|s-u|<\delta}\left|Y^{\prime}(s, \bullet)-Y^{\prime}(u, \bullet)\right|\right\|_{S^{p}[0, T]} \rightarrow 0 \text { as } \delta \downarrow 0 .
$$

If $0=t_{0} \leqslant t_{1} \leqslant \cdots \leqslant t_{m}=T, g_{0}, \ldots, g_{m}$ are defined as in the first paragraph of Section 3, and

$$
J(t, s):=\sum_{k=0}^{m-1} Y\left(t_{k}, s\right) g_{k}(t), \quad t, s \in[0, T]
$$


(i) $\mathcal{V}_{p}(Y,[0, T]) \leqslant T \sup _{t \in[0, T]}\left\|Y^{\prime}(t, \bullet)\right\|_{S^{p}[0, T]}$,

(ii) $J_{k}(s):=Y\left(t_{k}, s\right), s \in[0, T]$, is such that $J_{k} \in S^{p}[0, T]$ for all $k$, and $\mathcal{V}_{p}(J,[0, T]) \leqslant \mathcal{V}_{p}(Y,[0, T])$,

(iii)

$$
\mathcal{V}_{p}(J-Y,[0, T]) \leqslant(1+2 C) T \sup _{k=1, \ldots, m}\left\|\sup _{u, s \in\left[t_{k-1}, t_{k}\right]}\left|Y^{\prime}(u, \bullet)-Y^{\prime}(s, \bullet)\right|\right\|_{S^{p}[0, T]} .
$$

Proof. (i) Let $\left(\tau_{j}\right)_{j}$ be a partition. We may assume that $0=\tau_{0} \leqslant \tau_{1} \leqslant \cdots \leqslant \tau_{n}=T$. Then

$$
\begin{aligned}
& \sum_{j=0}^{n-1}\left\|Y\left(\tau_{j+1}, \bullet\right)-Y\left(\tau_{j}, \bullet\right)\right\|_{S^{p}} \\
& \leqslant \sum_{j=0}^{n-1}\left(\left\|Y^{\prime}\left(\tau_{j}, \bullet\right)\left(\tau_{j+1}-\tau_{j}\right)\right\|_{S^{p}}\right. \\
& \left.\quad+\left(\tau_{j+1}-\tau_{j}\right) C \sup _{u, s \in\left[\tau_{j}, \tau_{j+1}\right]}\left\|Y^{\prime}(s, \bullet)-Y^{\prime}(u, \bullet)\right\|_{S^{p}}\right) \\
& \quad \leqslant \sup _{t \in[0, T]}\left\|Y^{\prime}(t, \bullet)\right\|_{S^{p}} T+C T \sup _{j} \sup _{u, s \in\left[\tau_{j}, \tau_{j+1}\right]}\left\|Y^{\prime}(s, \bullet)-Y^{\prime}(u, \bullet)\right\|_{S^{p}} .
\end{aligned}
$$

Thus we obtain

$$
\mathcal{V}_{p}(Y,[0, T]) \leqslant T \sup _{t \in[0, T]}\left\|Y^{\prime}(t, \bullet)\right\|_{S^{p}}
$$

(ii) It follows directly from the assumptions at the begin of the section that $J_{k} \in S^{p}$. Next, let $\left(\tau_{\ell}\right)_{\ell=0}^{n}$ be a partition. We may assume that it is a refinement of $\left(t_{k}\right)_{k}$ and relabel such that

$$
t_{k-1}=\tau_{0}^{k} \leqslant \tau_{1}^{k} \leqslant \cdots \leqslant \tau_{j_{k}}^{k}=t_{k}, \quad k=1, \ldots, m .
$$

Then

$$
\begin{aligned}
\sum_{k=1}^{m} \sum_{j=1}^{j_{k}}\left\|J\left(\tau_{j}^{k}, \bullet\right)-J\left(\tau_{j-1}^{k}, \bullet\right)\right\|_{S^{p}} & \\
= & \sum_{k=1}^{m} \sum_{j=1}^{j_{k}} \| Y\left(t_{k-1}, \bullet\right) g_{k-1}\left(\tau_{j}^{k}\right)+Y\left(t_{k}, \bullet\right) g_{k}\left(\tau_{j}^{k}\right) \\
& \quad-\left(Y\left(t_{k-1}, \bullet\right) g_{k-1}\left(\tau_{j-1}^{k}\right)+Y\left(t_{k}, \bullet\right) g_{k}\left(\tau_{j-1}^{k}\right)\right) \|_{S^{p}} \\
= & \sum_{k=1}^{m} \sum_{j=1}^{j_{k}} \| Y\left(t_{k-1}, \bullet\right)+\left(Y\left(t_{k}, \bullet\right)-Y\left(t_{k-1}, \bullet\right)\right) g_{k}\left(\tau_{j}^{k}\right) \\
& \quad-\left(Y\left(t_{k-1}, \bullet\right)+\left(Y\left(t_{k}, \bullet\right)-Y\left(t_{k-1}, \bullet\right)\right) g_{k}\left(\tau_{j-1}^{k}\right)\right) \|_{S^{p}} \\
= & \sum_{k=1}^{m}\left\|Y\left(t_{k}, \bullet\right)-Y\left(t_{k-1}, \bullet\right)\right\|_{S^{p}} \leqslant \mathcal{V}_{p}(Y)
\end{aligned}
$$


(iii) With the notations of (ii), Lemma 4.1 yields

$$
\begin{array}{r}
\sum_{k=1}^{m} \sum_{j=1}^{j_{k}}\left\|J\left(\tau_{j}^{k}, \bullet\right)-Y\left(\tau_{j}^{k}, \bullet\right)-\left(J\left(\tau_{j-1}^{k}, \bullet\right)-Y\left(\tau_{j-1}^{k}, \bullet\right)\right)\right\|_{S^{p}} \\
\quad \leqslant(1+2 C) T \sup _{k=1, \ldots, m}\left\|\sup _{u, s \in[0, T]}\left|Y^{\prime}(u, \bullet)-Y^{\prime}(s, \bullet)\right|\right\|_{S^{p}},
\end{array}
$$

so

$$
\mathcal{V}_{p}(J-Y) \leqslant(1+2 C) T \sup _{k=1, \ldots, m}\left\|\sup _{u, s \in[0, T]}\left|Y^{\prime}(u, \bullet)-Y^{\prime}(s, \bullet)\right|\right\|_{S^{p}} .
$$

Lemma 4.3. Assume in addition to the assumption of Lemma 4.2 that $\sup _{t, s \in[0, T]}$ $|Y(t, s)| \in L^{p}$ and

$$
\left\|\sup _{|s-u|<\delta}|Y(s, \bullet)-Y(u, \bullet)|\right\|_{S^{p}[0, T]} \rightarrow 0 \quad \text { as } \delta \downarrow 0 .
$$

Choose a sequence of partitions

$$
0=t_{0}^{n} \leqslant t_{1}^{n} \leqslant \cdots \leqslant t_{m_{n}}^{n}=T
$$

such that $\sup _{k}\left|t_{k+1}^{n}-t_{k}^{n}\right| \rightarrow 0$ as $n \rightarrow \infty$, let $g_{k}^{n}$ be the corresponding functions as defined in the first paragraph of Section 3, and define

$$
Y^{n}(t, s):=\sum_{k=0}^{m_{n}-1} Y\left(t_{k}^{n}, s\right) g_{k}^{n}(t), \quad t, s \in[0, T], n \in \mathbb{N}
$$

Let $1 \leqslant q \leqslant \infty$ and $1 \leqslant r<\infty$ be such that $\frac{1}{p}+\frac{1}{q}=\frac{1}{r}$. Let $Z \in H^{q}[0, T]$. Then

(i) $\int_{0}^{\bullet} Y^{n}(\bullet, s-) \mathrm{d} Z(s) \in H^{r}[0, T]$ for all $n$,

(ii) for all $m, n \in \mathbb{N}$,

$$
\begin{aligned}
& \left\|\int_{0}^{\bullet} Y^{n}(\bullet, s-) \mathrm{d} Z(s)-\int_{0}^{\bullet} Y^{m}(\bullet, s-) \mathrm{d} Z(s)\right\|_{H^{r}[0, T]} \\
& \leqslant\left(\left\|\sup _{t, s \in[0, T]}\left|Y^{n}(t, s)-Y^{m}(t, s)\right|\right\|_{L^{p}}\right. \\
& \left.\quad+\left(1+c_{r}\right) \mathcal{V}_{p}\left(Y^{n}-Y^{m},[0, T]\right)\right)\|Z\|_{H^{q}[0, T]},
\end{aligned}
$$

(iii) $\left(\int_{0}^{\bullet} Y^{n}(\bullet, s-) \mathrm{d} Z(s)\right)_{n}$ is a Cauchy sequence in $H^{r}[0, T]$,

(iv) $\int_{0}^{t} Y^{n}(t, s-) \mathrm{d} Z(s) \rightarrow \int_{0}^{t} Y(t, s-) \mathrm{d} Z(s)$ in probability for all $t \in[0, T]$,

(v) $\int_{0}^{\bullet} Y(\bullet, s-) \mathrm{d} Z(s) \in H^{r}[0, T]$ and

$$
\int_{0}^{\bullet} Y^{n}(\bullet, s-) \mathrm{d} Z(s) \rightarrow \int_{0}^{\bullet} Y(\bullet, s-) \mathrm{d} Z(s) \text { in } H^{r}[0, T],
$$


(vi) for each $n$,

$$
\begin{aligned}
& \left\|\int_{0}^{\bullet} Y^{n}(\bullet, s-) \mathrm{d} Z(s)\right\|_{H^{r}[0, T]} \\
& \quad \leqslant\left(\left\|\sup _{t, s \in[0, T]}\left|Y^{n}(t, s)\right|\right\|_{L^{p}}+2\left(1+c_{r}\right) \mathcal{V}_{p}\left(Y^{n},[0, T]\right)\right)\|Z\|_{H^{q}[0, T]},
\end{aligned}
$$

(vii)

$$
\begin{aligned}
& \left\|\int_{0}^{\bullet} Y(\bullet, s-) \mathrm{d} Z(s)\right\|_{H^{r}[0, T]} \\
& \quad \leqslant\left(\left\|\sup _{t, s \in[0, T]} \mid Y(t, s)\right\| \|_{L^{p}}+2\left(1+c_{r}\right) \mathcal{V}_{p}(Y,[0, T])\right)\|Z\|_{H^{q}[0, T]} .
\end{aligned}
$$

Proof. (i) is contained in Lemma 3.5.

(ii) Notice that

$$
\int_{0}^{\bullet} Y^{n}(\bullet, s-) \mathrm{d} Z(s)-\int_{0}^{\bullet} Y^{m}(\bullet, s-) \mathrm{d} Z(s)=\int_{0}^{\bullet}\left(Y^{n}(\bullet, s-)-Y^{m}(\bullet, s-)\right) \mathrm{d} Z(s) .
$$

Recall that $\sup _{s, t \in[0, T]}\left|Y^{\prime}(t, s)\right| \in L^{p}$, so that $\sup _{t \in[0, T]}\left\|Y^{\prime}(t, \bullet)\right\|_{S^{p}}<\infty$, and apply Lemma 4.2(i)-(ii) and Corollary 3.6.

(iii) Let $n, \ell \in \mathbb{N}$ and let $\delta^{n, \ell}:=\sup _{k}\left|t_{k+1}^{n}-t_{k}^{n}\right| \vee \sup _{k}\left|t_{k+1}^{\ell}-t_{k}^{\ell}\right|$. If we choose an arbitrary $t \in\left[t_{k-1}^{n}, t_{k}\right]$ and $t \in\left[t_{j-1}^{\ell}, t_{j}^{\ell}\right]$, then

$$
\begin{aligned}
& \left|\sum_{k=0}^{m_{n}-1} Y\left(t_{k}^{n}, s\right) g_{k}^{n}(t)-\sum_{k=0}^{m_{\ell}-1} Y\left(t_{k}^{\ell}, s\right) g_{k}^{\ell}(t)\right| \\
& =\mid Y\left(t_{k-1}^{n}, s\right) g_{k-1}^{n}(t)+Y\left(t_{k}^{n}, s\right) g_{k}^{n}(t) \\
& \quad-\left(Y\left(t_{j-1}^{\ell}, s\right) g_{j-1}^{\ell}(t)-Y\left(t_{j}^{\ell}, s\right) g_{j}^{\ell}(t)\right) \mid \\
& =\mid Y\left(t_{k-1}^{n}, s\right)+\left(Y\left(t_{k}^{n}, s\right)-Y\left(t_{k-1}^{n}, s\right)\right) g_{k}^{n}(t) \\
& \quad-Y\left(t_{j-1}^{\ell}, s\right)-\left(Y\left(t_{j}^{\ell}, s\right)-Y\left(t_{j-1}^{\ell}, s\right)\right) g_{j}^{\ell}(t) \mid \\
& \leqslant\left|Y\left(t_{k-1}^{n}, s\right)-Y\left(t_{j-1}^{\ell}, s\right)\right|+\left|Y\left(t_{k}^{n}, s\right)-Y\left(t_{k-1}^{n}, s\right)\right| \\
& \quad+\left|Y\left(t_{j}^{\ell}, s\right)-Y\left(t_{j-1}^{\ell}, s\right)\right| \\
& \leqslant 3 \sup _{a, b \in\left[t-\delta^{n, \ell}, t+\delta^{n, \ell}\right]}|Y(a, s)-Y(b, s)| .
\end{aligned}
$$

Hence

$$
\begin{aligned}
& \left\|\sup _{t, s \in[0, T]}\left|Y^{n}(t, s)-Y^{\ell}(t, s)\right|\right\|_{L^{p}} \\
& \quad=\left\|\sup _{t, s \in[0, T]}\left|\sum_{k=0}^{m_{n}-1} Y\left(t_{k}^{n}, s\right) g_{k}^{n}(t)-\sum_{k=0}^{m_{\ell}-1} Y\left(t_{k}^{\ell}, s\right) g_{k}^{\ell}(t)\right|\right\|_{L^{p}} \\
& \quad \leqslant\left\|\sup _{t, s \in[0, T]} 3 \sup _{a, b \in\left[t-\delta^{n, \ell}, t+\delta^{n, \ell}\right]}|Y(a, s)-Y(b, s)|\right\|_{L^{p}} \\
& \quad \leqslant 3\left\|\sup _{s \in[0, T]|a-b| \leqslant 2 \delta^{n, \ell}}|Y(a, s)-Y(b, s)|\right\|_{L^{p}} \\
& \quad \rightarrow 0 \text { if } n, \ell \rightarrow \infty .
\end{aligned}
$$


Now the assertion follows from (ii) with aid of Lemma 4.2(iii) and the observation that

$$
\begin{aligned}
& \sup _{k=1, \ldots, m}\left\|\sup _{t_{k-1}^{n} \leqslant a, b \leqslant t_{k}^{n}}\left|Y^{\prime}(a, \bullet)-Y^{\prime}(b, \bullet)\right|\right\|_{S^{p}} \\
& \leqslant\left\|\sup _{k} \sup _{t_{k-1}^{n} \leqslant a, b \leqslant t_{k}^{n}} \mid Y^{\prime}(a, \bullet)-Y^{\prime}(b, \bullet)\right\|_{S^{p}} \\
& \leqslant\left\|\sup _{|a-b|<\delta^{n, \ell}}\left|Y^{\prime}(a, \bullet)-Y^{\prime}(b, \bullet)\right|\right\|_{S^{p}},
\end{aligned}
$$

so that $\mathcal{V}_{p}\left(Y^{n}-Y\right) \rightarrow 0$ and $\mathcal{V}_{p}\left(Y^{\ell}-Y\right) \rightarrow 0$ as $n, \ell \rightarrow \infty$ and therefore $\mathcal{V}_{p}\left(Y^{n}-\right.$ $\left.Y^{\ell}\right) \rightarrow 0$ as $n, \ell \rightarrow \infty$.

(iv) Let $\delta^{n}:=\sup _{k}\left|t_{k+1}^{n}-t_{k}^{n}\right|$. For each $t \in[0, T]$,

$$
\begin{aligned}
& \left\|\sup _{s \in[0, T]}\left|Y^{n}(t, s)-Y(t, s)\right|\right\|_{L^{p}} \leqslant \| \sup _{s \in[0, T]|a-b|<\delta^{n}} \sup _{\mid a, s)-Y(b, s) \mid} \mid Y\left(a, \|_{L^{p}}\right. \\
& =\left\|\sup _{|a-b|<\delta^{n}}|Y(a, \bullet)-Y(b, \bullet)|\right\|_{S^{p}} \rightarrow 0 \text { as } n \rightarrow \infty,
\end{aligned}
$$

hence $\sup _{s \in[0, T]}\left|Y^{n}(t, s)-Y(t, s)\right| \rightarrow 0$ in probability as $n \rightarrow \infty$. Therefore by Theorem 2.5, for each $\tau \in[0, T]$,

$$
\sup _{0 \leqslant s \leqslant \tau}\left|\int_{0}^{s} Y^{n}(t, \sigma-) \mathrm{d} Z(\sigma)-\int_{0}^{s} Y(t, \sigma-) \mathrm{d} Z(\sigma)\right| \rightarrow 0 \text { in probability. }
$$

(v) By (iii) and the completeness of $H^{r}$ (Theorem 2.1), there is an $H \in H^{r}$ such that

Since for any $t \in[0, T]$,

$$
\left\|\int_{0}^{\bullet} Y^{n}(\bullet, s-) \mathrm{d} Z(s)-H(\bullet)\right\|_{H^{r}} \rightarrow 0, \quad n \rightarrow \infty .
$$

$$
\begin{aligned}
& \left\|\int_{0}^{t} Y^{n}(t, s-) d Z(s)-H(t)\right\|_{L^{r}} \\
& \quad \leqslant\left\|\int_{0}^{\bullet} Y^{n}(\bullet, s-) \mathrm{d} Z(s)-H(\bullet)\right\|_{S^{r}} \\
& \quad \leqslant c_{r}\left\|\int_{0}^{\bullet} Y^{n}(\bullet, s-) \mathrm{d} Z(s)-H(\bullet)\right\|_{H^{r}} \rightarrow 0 \text { as } n \rightarrow \infty,
\end{aligned}
$$

item (iv) yields that $\int_{0}^{t} Y(t, s-) \mathrm{d} Z(s)=H(t)$ a.s. Hence

$$
\int_{0}^{t} Y(t, s-) \mathrm{d} Z(s)=H(t) \text { for all } t \in[0, T] \text { a.s. }
$$

(vi) is contained in Corollary 3.6.

(vii) By (v) and (vi) and the arguments in the proof of (iii) we have

$$
\begin{array}{r}
\left\|\int_{0}^{\bullet} Y(\bullet, s-) \mathrm{d} Z(s)\right\|_{H^{r}}=\lim _{n \rightarrow \infty}\left\|\int_{0}^{\bullet} Y^{n}(\bullet, s-) \mathrm{d} Z(s)\right\|_{H^{r}} \\
\leqslant\left(\left\|\sup _{t, s \in[0, T]}|Y(t, s)|\right\|_{L^{p}}+2\left(1+c_{r}\right) \mathcal{V}_{p}(Y)\right)\|Z\|_{H^{q}} .
\end{array}
$$

Observe that (v) and (vii) of Lemma 4.3 together establish Theorem 1.4. 


\section{Application to convolutions}

This sections concerns an Émery inequality for convolutions of the form

$$
\int_{0}^{t} g(t-s) Y(s-) \mathrm{d} Z(s), \quad t \in[0, T]
$$

where $g$ is a deterministic function, $Y$ an adapted càdlàg process, and $Z$ a semimartingale. If the function $g$ is right continuous, then the integral is well defined. We will first study continuously differentiable functions $g$ by means of Theorem 1.4, then study pure jump functions, and then combine the results to prove Theorem 1.2 .

Lemma 5.1. Let $1 \leqslant p \leqslant \infty, 1 \leqslant q \leqslant \infty$, and $1 \leqslant r<\infty$ be such that $\frac{1}{p}+\frac{1}{q}=\frac{1}{r}$. Let $T>0$ and let $g:[0, T] \rightarrow \mathbb{R}$ be continuously differentiable. Then for every $Z \in H^{q}=H^{q}[0, T]$ and every $Y \in S^{p}=S^{p}[0, T]$ one has $\int_{0}^{\bullet} g(\bullet-s) Y(s-) \mathrm{d} Z(s) \in$ $H^{r}=H^{r}[0, T]$ and

$$
\begin{aligned}
& \left\|\int_{0}^{\bullet} g(\bullet-s) Y(s-) \mathrm{d} Z(s)\right\|_{H^{r}} \\
& \quad \leqslant\left(\|g\|_{\infty}+2\left(1+c_{r}\right) T\left\|g^{\prime}\right\|_{\infty}\right)\|Y\|_{S^{p}}\|Z\|_{H^{q}} .
\end{aligned}
$$

Proof. Let $\eta>0$ be arbitrarily small. We begin by choosing a function $g_{0}$ : $(-\infty, T] \rightarrow \mathbb{R}$ such that

$$
\begin{aligned}
& g_{0}(t)=g(t), \quad 0 \leqslant t \leqslant T, \\
& g_{0} \text { is constant on }(-\infty,-1], \\
& g_{0} \text { is continuously differentiable, } \\
& \left\|g_{0}^{\prime}\right\|_{\infty}<\left\|g^{\prime}\right\|_{\infty}+\eta \\
& \left\|g_{0}\right\|_{\infty}<\|g\|_{\infty}+\eta
\end{aligned}
$$

Observe that

$$
\int_{0}^{\bullet} g(\bullet-s) Y(s-) \mathrm{d} Z(s)=\int_{0}^{\bullet} g_{0}(\bullet-s) Y(s-) \mathrm{d} Z(s) .
$$

Let

$$
Y(t, s):=g_{0}(t-s) Y(s) \quad \text { and } \quad Y^{\prime}(t, s):=g_{0}^{\prime}(t-s) Y(s), \quad t, s \in[0, T] .
$$

Then for each $t \geqslant 0, Y(t, \bullet) \in S^{p}$ and $\left\|\sup _{s, t \in[0, T]} \mid Y(t, s)\right\|_{L^{p}} \leqslant\left\|g_{0}\right\|_{\infty}\|Y\|_{S^{p}}$. Further, $Y^{\prime}(t, \bullet) \in S^{p}$ for all $t \in[0, T]$ and $\left\|\sup _{s, t \in[0, T]}\left|Y^{\prime}(t, s)\right|\right\|_{L^{p}} \leqslant\left\|g_{0}^{\prime}\right\|_{\infty}\|Y\|_{S^{p}}$. Moreover, for any $0 \leqslant t \leqslant \tau \leqslant T$ and a suitable choice of $\theta_{\tau, t, s} \in[t, \tau]$, we have

$$
\begin{aligned}
\left\|Y(\tau, \bullet)-Y(t, \bullet)-(\tau-t) Y^{\prime}(t, \bullet)\right\|_{S^{p}} \\
=\left\|\sup _{s \in[0, T]}\left|g_{0}(\tau-s)-g_{0}(t-s)-g_{0}^{\prime}(t-s)(\tau-t)\right| Y(s) \mid\right\|_{L^{p}} \\
=\left\|\sup _{s \in[0, T]}\left|g_{0}^{\prime}\left(\theta_{\tau, t, s}-s\right)-g_{0}^{\prime}(t-s)\right|(\tau-t) Y(s)\right\|_{L^{p}} \\
=\left\|\sup _{s \in[0, T]}\left|Y^{\prime}\left(\theta_{\tau, t, s}, s\right)-Y^{\prime}(t, s)\right|\right\|_{L^{p}}(\tau-t) \\
\leqslant\left\|\sup _{s \in[0, T]} \sup _{t \leqslant \theta \leqslant \tau}\left|Y^{\prime}(\theta, s)-Y^{\prime}(t, s)\right|\right\|_{L^{p}}(\tau-t) \\
\leqslant(\tau-t)\left\|\sup _{t \leqslant \theta \leqslant \tau} \mid Y^{\prime}(\theta, \bullet)-Y^{\prime}(t, \bullet)\right\|_{S^{p}} \cdot
\end{aligned}
$$


Moreover,

$$
\begin{aligned}
& \left\|\sup _{|\tau-\theta|<\delta}\left|Y^{\prime}(\tau, \bullet)-Y^{\prime}(\theta, \bullet)\right|\right\|_{S^{p}} \\
& \leqslant\left\|\sup _{|\tau-\theta|<\delta}\left|g_{0}^{\prime}(\tau)-g_{0}^{\prime}(\theta)\right| \sup _{s \in[0, T]}|Y(s)|\right\|_{L^{p}} \\
& \leqslant \sup _{|\tau-\theta|<\delta}\left|g_{0}^{\prime}(\tau)-g_{0}^{\prime}(\theta)\right|\|Y\|_{S^{p}} \rightarrow 0 \text { as } \delta \downarrow 0,
\end{aligned}
$$

because $g_{0}^{\prime}$ is uniformly continuous. Similarly, $\left\|\sup _{|\tau-\theta|<\delta}|Y(\tau, \bullet)-Y(\theta, \bullet)|\right\|_{S^{p}} \rightarrow 0$ as $\delta \downarrow 0$. Finally, we estimate

$$
\begin{gathered}
\mathcal{V}_{p}(Y,[0, T])=\sup \left\{\sum_{j=0}^{m-1}\left\|\sup _{s \in[0, T]}\left|g_{0}\left(\tau_{j+1}-s\right) Y(s)-g_{0}\left(\tau_{j}-s\right) Y(s)\right|\right\|_{L^{p}}:\right. \\
\left.0 \leqslant \tau_{0} \leqslant \cdots \leqslant \tau_{m} \leqslant T, m \in \mathbb{N}\right\} \leqslant T\|Y\|_{S^{p}}\left\|g_{0}^{\prime}\right\|_{\infty} .
\end{gathered}
$$

Thus we can apply Theorem 1.4 and obtain $\int_{0}^{\bullet} g_{0}(\bullet-s) Y(s-) \mathrm{d} Z(s) \in H^{r}$ and

$$
\begin{aligned}
& \left\|\int_{0}^{\bullet} Y(\bullet, s-) \mathrm{d} Z(s)\right\|_{H^{r}} \\
& \quad \leqslant\left(\left\|\sup _{t, s \in[0, T]}|Y(t, s)|\right\|_{L^{p}}+2\left(1+c_{r}\right) \mathcal{V}_{p}(Y)\right)\|Z\|_{H^{q}} \\
& \quad \leqslant\left(\left\|g_{0}\right\|_{\infty}\|Y\|_{S^{p}}+2\left(1+c_{r}\right) T\left\|g_{0}^{\prime}\right\|_{\infty}\|Y\|_{S^{p}}\right)\|Z\|_{H^{q}} \\
& \quad \leqslant\left(\|g\|_{\infty}+\eta+2\left(1+c_{r}\right) T\left(\left\|g^{\prime}\right\|_{\infty}+\eta\right)\right)\|Y\|_{S^{p}}\|Z\|_{H^{q}} .
\end{aligned}
$$

We infer that

$$
\begin{aligned}
& \left\|\int_{0}^{\bullet} g(\bullet-s) Y(s-) \mathrm{d} Z(s)\right\|_{H^{r}} \\
& \quad \leqslant\left(\|g\|_{\infty}+2\left(1+c_{r}\right) T\left\|g^{\prime}\right\|_{\infty}\right)\|Y\|_{S^{p}}\|Z\|_{H^{q}}
\end{aligned}
$$

Let us next extend the scope to functions that have a derivative that is of bounded variation. We use a decomposition into a $C^{1}$-function and a so-called pure jump function.

For an interval $I$, let $B V(I)$ denote the vector space of functions $f: I \rightarrow \mathbb{R}$ that have finite total variation over $I$. For each $f \in B V(I)$ the left and right hand limits $f(x-)$ and $f(x+)$ exist at every $x$ in the interior of $I$. Moreover, $f(a+)$ exists if $I$ has a left endpoint $a$ and $f(b-)$ exists if $I$ has a right endpoint $b$. There are at most countably many points $x$ in $I$ where $f(x-) \neq f(x+)$, so each $B V$ function is a.e. equal to a càdlàg function. For a càdlàg function $f \in B V(I)$ the sum $\sum_{x \in I}|(\Delta f)(x)|$ is finite and we call the function

$$
g(x):=\sum_{y \in I}(\Delta g)(y) 1_{[y, \infty) \cap I}(x), \quad x \in I,
$$


the pure jump part of $f$. Notice that $f-g$ is continuous on $I$. Let $B V^{1}[a, b]$ denote the vector space of indefinite integrals of functions in $B V[a, b]$, that is

$$
B V^{1}[a, b]=\left\{f:[a, b] \rightarrow \mathbb{R}: \exists g \in B V[a, b] \text { such that } f(x)=f(a)+\int_{a}^{x} g(y) \mathrm{d} y\right\} .
$$

In other words, $B V^{1}[a, b]$ consists of the absolutely continuous functions with a derivative that is almost everywhere equal to a function of bounded variation. For $f \in B V^{1}[a, b]$ we let $f^{\prime}$ denote the unique càdlàg function in $B V[a, b)$ with $f(x)=$ $f(a)+\int_{a}^{x} f^{\prime}(y) \mathrm{d} y$ for all $x \in[a, b]$, and set for convenience $f^{\prime}(b):=f^{\prime}(b-)$.

A càdlàg function $g:[0, T] \rightarrow \mathbb{R}$ of the form

$$
g(t)=\sum_{j=1}^{\infty} \alpha_{j} 1_{\left[t_{j}, T\right]}(t), \quad t \in[0, T]
$$

will be called a pure jump function and it is of bounded variation if $\sum_{j}\left|\alpha_{j}\right|<\infty$. The vector space of càdlàg pure jump functions of bounded variation on $[0, T]$ will be denoted by $P J B V[0, T]$.

The next lemma concerns the jump parts.

Lemma 5.2. Let $1 \leqslant p \leqslant \infty, 1 \leqslant q \leqslant \infty$, and $1 \leqslant r<\infty$ be such that $\frac{1}{p}+\frac{1}{q}=\frac{1}{r}$. Let $T>0$ and let $g:[0, T] \rightarrow \mathbb{R}$ be given by

$$
g(t)=\sum_{j=1}^{\infty} 1_{\left[t_{j}, T\right]}(t)\left(\alpha_{j}\left(t-t_{j}\right)+\beta_{j}\right), \quad t \in[0, T],
$$

where $t_{j} \in[0, T]$ and $\alpha_{j}, \beta_{j} \in \mathbb{R}, j \in \mathbb{N}$, are such that $\sum_{j=1}^{\infty}\left|\alpha_{j}\right|<\infty$ and $\sum_{j=1}^{\infty}\left|\beta_{j}\right|<\infty$. Then for every $Y \in S^{p}[0, T]$ and every $Z \in H^{q}[0, T]$ one has

$$
\begin{aligned}
& \left\|\int_{0}^{\bullet} g(\bullet-s) Y(s-) \mathrm{d} Z(s)\right\|_{H^{r}[0, T]} \\
& \quad \leqslant \sum_{j=1}^{\infty}\left(\left|\beta_{j}\right|+\left|\alpha_{j}\right| T\left(2+c_{r}\right)\right)\|Y\|_{S^{p}[0, T]}\|Z\|_{H^{q}[0, T]} .
\end{aligned}
$$

Proof. Let $Y \in S^{p}=S^{p}[0, T]$ and $Z \in H^{q}=H^{q}[0, T]$. First, let $n \in \mathbb{N}$ and assume that $\alpha_{j}=0$ and $\beta_{j}=0$ for all $j>m$. We have for $t \in[0, T]$,

$$
\begin{aligned}
\int_{0}^{t} g(t-s) Y(s-) \mathrm{d} Z(s) \\
\quad=\sum_{j=1}^{m} \int_{0}^{t} 1_{\left[t_{j}, \infty\right)}(t-s)\left(\alpha_{j}\left(t-s-t_{j}\right)+\beta_{j}\right) Y(s-) \mathrm{d} Z(s) \\
\quad=\sum_{j=1}^{m} \int_{0}^{\infty} 1_{\left[0, t-t_{j}\right]}(s)\left(\alpha_{j}\left(t-s-t_{j}\right)+\beta_{j}\right) Y(s-) \mathrm{d} Z(s) \\
\quad=\sum_{j=1}^{m}\left(\alpha_{j}\left(t-t_{j}\right) \int_{0}^{\left(t-t_{j}\right)^{+}} Y(s-) \mathrm{d} Z(s)+\int_{0}^{\left(t-t_{j}\right)^{+}}\left(\beta_{j}-\alpha_{j} s\right) Y(s-) \mathrm{d} Z(s)\right) .
\end{aligned}
$$

The latter term is easily estimated by Émery's inequality. We obtain

$$
\sum_{j=1}^{m} \int_{0}^{\left(\bullet-t_{j}\right)^{+}}\left(\beta_{j}-\alpha_{j} s\right) Y(s-) \mathrm{d} Z(s) \in H^{r}
$$


and

$$
\begin{aligned}
& \left\|\sum_{j=1}^{m} \int_{0}^{\left(\bullet-t_{j}\right)^{+}}\left(\beta_{j}-\alpha_{j} s\right) Y(s-) \mathrm{d} Z(s)\right\|_{H^{r}} \\
& \quad \leqslant \sum_{j=1}^{m}\left\|s \mapsto\left(\beta_{j}-\alpha_{j} s\right) Y(s)\right\|_{S^{p}}\|Z\|_{H^{q}} \\
& \quad \leqslant \sum_{j=1}^{m}\left(\left|\beta_{j}\right|+\left|\alpha_{j}\right| T\right)\|Y\|_{S^{p}}\|Z\|_{H^{q}} .
\end{aligned}
$$

Denote

$$
V(t):=\int_{0}^{t} Y(s-) \mathrm{d} Z(s), \quad t \in[0, T],
$$

and let $V(t)=V(T)$ for $t>T$. We want to show that $(t V(t))_{t \geqslant 0}$ is a semimartingale and estimate its $H^{r}$-norm. We have that $V$ is a semimartingale. Let $B$ be an FVprocess and $M$ a local martingale such that

$$
V=B+M
$$

and denote $A(t):=t \wedge T, t \geqslant 0$. According to Theorem 2.7, the product $M A$ decomposes into

$$
(M A)(t)=\int_{(0, t]} M(s) \mathrm{d} A(s)+\int_{(0, t]} A(s-) \mathrm{d} M(s),
$$

where the former integral is an FV-process and the latter a local martingale. Theorem 2.4 yields that

$$
\left[\int_{[0, \bullet]} s \wedge T \mathrm{~d} M(s), \int_{[0, \bullet]} s \wedge T \mathrm{~d} M(s)\right](t)=\int_{0}^{t}(s \wedge T)^{2} \mathrm{~d}[M, M](s) \leqslant T^{2}[M, M](T),
$$

$t \geqslant 0$, and according to Theorem 2.3(e),

$$
\begin{aligned}
\operatorname{Var}_{[0, \infty)}\left(\int_{0}^{\bullet} M(s) \mathrm{d}(s \wedge T)\right) & \leqslant \sup _{t \geqslant 0}|M(t)| \operatorname{Var}_{[0, \infty)}(s \wedge T) \\
& =T \sup _{t \geqslant 0}|M(t)| .
\end{aligned}
$$

Hence for the product $V A$ we have

$$
\begin{aligned}
& \|(V A)\|_{H^{r}} \leqslant\left\|[A \bullet M, A \bullet M]_{\infty}^{1 / 2}+\operatorname{Var}_{[0, \infty)}(M \bullet A+B A)\right\|_{L^{r}} \\
& \quad \leqslant\left\|T[M, M]_{T}^{1 / 2}+T \sup _{t \geqslant 0}|M(t)|+T \operatorname{Var}_{[0, \infty)}(B)\right\|_{L^{r}} \\
& \quad \leqslant T\left\|[M, M]_{\infty}^{1 / 2}+\operatorname{Var}_{[0, \infty)}(B)\right\|_{L^{r}}+T\|M\|_{S^{r}} \\
& \quad \leqslant T\left\|[M, M]_{\infty}^{1 / 2}+\operatorname{Var}_{[0, \infty)}(B)\right\|_{L^{r}}+T c_{r}\left\|[M, M]_{\infty}^{1 / 2}\right\|_{L^{r}},
\end{aligned}
$$

where the latter inequality is due to Theorem 2.1-2.2. So $\|V A\|_{H^{r}} \leqslant T(1+$ 
$\left.c_{r}\right)\|V\|_{H^{r}}$. It follows that

$$
\begin{aligned}
& \left\|\sum_{j=1}^{m} \alpha_{j}\left(\bullet-t_{j}\right) \int_{0}^{\left(\bullet-t_{j}\right)^{+}} Y(s-) \mathrm{d} Z(s)\right\|_{H^{r}[0, T]} \\
& \quad \leqslant \sum_{j=1}^{m}\left|\alpha_{j}\right|\left\|(A V)^{T}\right\|_{H^{r}[0, T]} \\
& \quad \leqslant \sum_{j=1}^{m}\left|\alpha_{j}\right| T\left(1+c_{r}\right)\left\|\int_{0}^{\bullet} Y(s-) \mathrm{d} Z(s)\right\|_{H^{r}[0, T]} \\
& \quad \leqslant\left(\sum_{j=1}^{m}\left|\alpha_{j}\right| T\left(1+c_{r}\right)\right)\|Y\|_{S^{p}}\|Z\|_{H^{q}} .
\end{aligned}
$$

We infer that $\int_{0}^{\bullet} g(\bullet-s) Y(s-) \mathrm{d} Z(s) \in H^{r}$ and

$$
\left\|\int_{0}^{\bullet} g(\bullet-s) Y(s-) \mathrm{d} Z(s)\right\|_{H^{r}} \leqslant \sum_{j=1}^{m}\left(\left|\beta_{j}\right|+\left|\alpha_{j}\right| T\left(2+c_{r}\right)\right)\|Y\|_{S^{p}}\|Z\|_{H^{q}} .
$$

Next we consider the general case. Observe that $g$ is a càdlàg function. Let

$$
g_{m}(t):=\sum_{j=1}^{m} 1_{\left[t_{j}, T\right]}(t)\left(\alpha_{j}\left(t-t_{j}\right)+\beta_{j}\right), \quad t \in[0, T], m \in \mathbb{N} .
$$

Then for $n>m$,

$$
\begin{aligned}
& \left\|\int_{0}^{\bullet} g_{n}(\bullet-s) Y(s-) \mathrm{d} Z(s)-\int_{0}^{\bullet} g_{m}(\bullet-s) Y(s-) \mathrm{d} Z(s)\right\|_{H^{r}} \\
& \leqslant \sum_{j=m+1}^{n}\left(\left|\beta_{j}\right|+\left|\alpha_{j}\right| T\left(2+c_{r}\right)\right)\|Y\|_{S^{p}}\|Z\|_{H^{q}} .
\end{aligned}
$$

As $H^{r}$ is complete (Theorem 2.1), it follows that $\int_{0}^{\bullet} g_{n}(\bullet-s) Y(s-) \mathrm{d} Z(s)$ converges in $H^{r}$ to some $H \in H^{r}$. For fixed $t \in[0, T], \sup _{0 \leqslant s \leqslant t}|Y(s)| \in L^{p}$, so

$$
\sup _{0 \leqslant s \leqslant t}\left|g_{m}(t-s) Y(s)-g(t-s) Y(s)\right| \leqslant\left\|g_{m}-g\right\|_{\infty} \sup _{0 \leqslant s \leqslant t}|Y(s)| \rightarrow 0
$$

in probability as $m \rightarrow \infty$. Hence by Theorem 2.5,

$$
\sup _{0 \leqslant s \leqslant t}\left|\int_{0}^{s} g_{m}(t-u) Y(u-) \mathrm{d} Z(u)-\int_{0}^{s} g(t-u) Y(u-) \mathrm{d} Z(u)\right| \rightarrow 0
$$

in probability. In particular,

$$
\int_{0}^{t} g_{m}(t-u) Y(u-) \mathrm{d} Z(u) \rightarrow \int_{0}^{t} g(t-u) Y(u-) \mathrm{d} Z(u)
$$

in probability. It follows that $H(t)=\int_{0}^{t} g(t-u) Y(u-) \mathrm{d} Z(u)$ and

$$
\begin{aligned}
& \left\|\int_{0}^{\bullet} g(\bullet-u) Y(u-) \mathrm{d} Z(u)\right\|_{H^{r}} \\
& =\lim _{m \rightarrow \infty}\left\|\int_{0}^{\bullet} g_{m}(\bullet-u) Y(u-) \mathrm{d} Z(u)\right\|_{H^{r}} \\
& \leqslant \sum_{j=1}^{\infty}\left(\left|\beta_{j}\right|+\left|\alpha_{j}\right| T\left(2+c_{r}\right)\right)\|Y\|_{S^{p}}\|Z\|_{H^{q}} .
\end{aligned}
$$


Theorem 5.3. Let $1 \leqslant p \leqslant \infty, 1 \leqslant q \leqslant \infty$, and $1 \leqslant r<\infty$ be such that $\frac{1}{p}+\frac{1}{q}=\frac{1}{r}$. Let $T>0$. If $g \in C^{1}[0, T]+B V^{1}[0, T]+P J B V[0, T]$, then for every $Y \in S^{p}=S^{p}[0, T]$ and every $Z \in H^{q}=H^{q}[0, T]$ one has $\int_{0}^{\bullet} g(\bullet-s) Y(s-) \mathrm{d} Z(s) \in$ $H^{r}=H^{r}[0, T]$ and

$$
\begin{aligned}
\left\|\int_{0}^{\bullet} g(\bullet-s) Y(s-) \mathrm{d} Z(s)\right\|_{H^{r}} & \leqslant\left(\left\|g_{1}\right\|_{\infty}+2\left(1+c_{r}\right)\left\|g_{1}^{\prime}\right\|_{\infty}\right. \\
& \left.+\left(2+c_{r}\right) T \operatorname{Var}_{[0, T]}\left(g_{2}^{\prime}\right)+\operatorname{Var}_{[0, T]}\left(g_{3}\right)\right)\|Y\|_{S^{p}}\|Z\|_{H^{q}} .
\end{aligned}
$$

Here $g=g_{1}+g_{2}+g_{3}$, where $g_{3} \in P J B V[0, T]$ is the pure jump part of $g, g_{2}^{\prime}$ is the pure jump part of $\left(g-g_{3}\right)^{\prime}, g_{2} \in B V^{1}[0, T]$ is the antiderivative of $g_{2}^{\prime}$ with $g_{2}(0)=0$, and $g_{1}=g-g_{2}-g_{3} \in C^{1}[0, T]$.

Proof. Let $g_{3}$ be the pure jump part of $g$. Then $g-g_{3} \in C^{1}[0, T]+B V^{1}[0, T]$. Let

$$
h(t):=\sum_{s \in[0, T]}\left(\Delta\left(g-g_{3}\right)^{\prime}\right)(s) 1_{[s, T]}(t), \quad t \in[0, T] .
$$

Choose $t_{j} \in[0, T]$ and $\alpha_{j}, \beta_{j} \in \mathbb{R}, j \in \mathbb{N}$, such that

$$
h(t)=\sum_{j=1}^{\infty} \alpha_{j} 1_{\left[t_{j}, T\right]}(t), \quad g_{3}(t)=\sum_{j=1}^{\infty} \beta_{j} 1_{\left[t_{j}, T\right]}(t), \quad t \in[0, T],
$$

and $\sum_{j=1}^{\infty}\left|\alpha_{j}\right|=\operatorname{Var}_{[0, T]}(h)<\infty, \sum_{j=1}^{\infty}\left|\beta_{j}\right|=\operatorname{Var}_{[0, T]}\left(g_{3}\right)<\infty$. Let

$$
g_{2}(t):=\int_{0}^{t} h(s) \mathrm{d} s=\sum_{j=1}^{\infty} 1_{\left[t_{j}, T\right]}(t) \alpha_{j}\left(t-t_{j}\right), \quad t \in[0, T] .
$$

As $\left(g-g_{3}\right)^{\prime}-g_{2}^{\prime} \in C[0, T]$, we have $g:=g_{1}-g_{3}-g_{2} \in C^{1}[0, T]$. If we apply Lemma 5.1 to $g_{1}$ and Lemma 5.2 to $g_{2}+g_{3}$, we arrive at the conclusion of the theorem.

\section{Existence for equations of variation-of-constants type}

In this section we will exploit the extended Émery inequality to show existence and uniquenss for stochastic equations of variation-of-constants type. We follow the proof of existence and uniqueness for stochastic differential equations as given in $[9]$.

Definition 6.1. Let $I \subset[0, \infty)$ be an interval. A map $F: \mathbb{D}(I) \rightarrow \mathbb{D}(I)$ is called functional Lipschitz if there exists an increasing (finite) process $(K(t))_{t \in I}$ such that for all $X, Y \in \mathbb{D}(I)$,

(i) $X^{T-}=Y^{T-} \Longrightarrow F(X)^{T-}=F(Y)^{T-}$ for every stopping time $T$,

(ii) $|F(X)(t)-F(Y)(t)| \leqslant K(t) \sup _{s \in I \cap[0, t]}|X(s)-Y(s)|$ a.s. for all $t \in I$.

Equalities of processes such as in (i) are meant up to indistinguishability. It is contained in (ii) that a functionally Lipschitz map $F$ is well-defined this way. Indeed, if $X$ and $Y$ in $\mathbb{D}(I)$ are indistinguishable, then (ii) yields that $F(X)(t)=F(Y)(t)$ a.s. for all $t \in I$ and hence $F(X)$ and $F(Y)$ are indistinguishable. 
Given constants $1 \leqslant p<\infty, t_{0}>0$, and $R>0$, we will use the following property of a function $g:\left[0, t_{0}\right] \rightarrow \mathbb{R}$ :

$g$ is càdlàg and for every $Y \in S^{p}\left[0, t_{0}\right]$ and $Z \in H^{\infty}\left[0, t_{0}\right]$ we have

$\int_{0}^{\bullet} g(\bullet-s) Y(s-) \mathrm{d} Z(s) \in H^{p}\left[0, t_{0}\right]$ and

$\left\|\int_{0}^{\bullet} g(\bullet-s) Y(s-) \mathrm{d} Z(s)\right\|_{H^{p}\left[0, t_{0}\right]} \leqslant R\|Y\|_{S^{p}\left[0, t_{0}\right]}\|Z\|_{H^{\infty}\left[0, t_{0}\right]}$.

We will establish Theorem 1.3 by a sequence of lemmas.

Lemma 6.2. Let $1 \leqslant p<\infty$, let $t_{0}>0$, and let $J \in S^{p}\left[0, t_{0}\right]$. Let $F$ : $\mathbb{D}\left[0, t_{0}\right] \rightarrow \mathbb{D}\left[0, t_{0}\right]$ be functional Lipschitz as in Definition 6.1 with $F(0)=0$ and $\sup _{t \in\left[0, t_{0}\right]}|K(t)| \leqslant k$ a.s. for some constant $k$. Let $g:\left[0, t_{0}\right] \rightarrow \mathbb{R}$ be a function and $R>0$ be a constant such that (6.1) is satisfied. Let $Z \in H^{\infty}\left[0, t_{0}\right]$ be such that $\|Z\|_{H \infty\left[0, t_{0}\right]} \leqslant 1 / 2 \gamma$, where

$$
\gamma=c_{p} k R
$$

Let $T$ be a stopping time. Then the equation

$$
X(t)=J^{T-}(t)+\left(\int_{0}^{\bullet} g(\bullet-s) F(X)(s-) \mathrm{d} Z(s)\right)^{T-}(t), \quad 0 \leqslant t \leqslant t_{0},
$$

has a unique solution $X$ in $S^{p}\left[0, t_{0}\right]$ and

$$
\|X\|_{S^{p}\left[0, t_{0}\right]} \leqslant 2\|J\|_{S^{p}\left[0, t_{0}\right]} .
$$

Proof. Define

$$
\Lambda(X)(t):=J(t)+\int_{0}^{t} g(t-s) F(X)(s-) \mathrm{d} Z(s), \quad t \in\left[0, t_{0}\right], X \in S^{p}\left[0, t_{0}\right] .
$$

By the assumption (6.1), the assumption $F(0)=0$, and the fact that $S^{p} \subset H^{p}$ we have that $\Lambda(X) \in S^{p}$ for every $X \in S^{p}$. Further, for $X, Y \in S^{p}$ we have

$$
\Lambda(X)-\Lambda(Y)=\int_{0}^{\bullet} g(\bullet-s)(F(X)(s-)-F(Y)(s-)) \mathrm{d} Z(s) \in H^{p} .
$$

Moreover,

$$
\begin{aligned}
& \left\|\Lambda(X)^{T-}-\Lambda(Y)^{T-}\right\|_{H^{p}} \leqslant\|\Lambda(X)-\Lambda(Y)\|_{H^{p}} \\
& \quad \leqslant R\|F(X)-F(Y)\|_{S^{p}}\|Z\|_{H^{\infty}} \\
& \quad \leqslant \frac{1}{2 c_{p} k} k\|X-Y\|_{S^{p}}
\end{aligned}
$$

so

$$
\left\|\Lambda(X)^{T-}-\Lambda(Y)^{T-}\right\|_{S^{p}} \leqslant c_{p}\left\|\Lambda(X)^{T-}-\Lambda(Y)^{T-}\right\|_{H^{p}} \leqslant \frac{1}{2}\|X-Y\|_{S^{p}} .
$$

Since $S^{p}$ is complete, we find a unique fixed point $X \in S^{p}$ of $\Lambda(\bullet)^{T-}$. This $X$ is the solution as asserted, and

$$
\|X-J\|=\left\|\Lambda(X)^{T-}-\Lambda(0)^{T-}\right\|_{S^{p}} \leqslant \frac{1}{2}\|X\|_{S^{p}},
$$

so that

$$
\|X\|_{S^{p}} \leqslant\|X-J\|_{S^{p}}+\|J\|_{S^{p}} \leqslant \frac{1}{2}\|X\|_{S^{p}}+\|J\|_{S^{p}}
$$

and hence

$$
\|X\|_{S^{p}} \leqslant 2\|J\|_{S^{p}}
$$


Definition 6.3. (see $[9$, p.192]) Let $I=[0, \infty)$ or $I=[a, b]$ for some $0 \leqslant a \leqslant b$. Let $Z \in H^{\infty}(I)$, and $\alpha>0$. Then $Z$ is called $\alpha$-sliceable, denoted by $Z \in \mathcal{S}(\alpha)$, if there exist stopping times $0=T_{0} \leqslant T_{1} \leqslant \cdots \leqslant T_{k}$ such that $Z=Z^{T_{k}-}$ and $\left\|\left(Z-Z^{T_{i}}\right)^{T_{i+1}-}\right\|_{H^{\infty}(I)} \leqslant \alpha$ for $i=0, \ldots, k-1$.

Theorem 6.4. (see $[9$, Theorem V.5, p.192]) Let $Z \in \mathbb{D}[0, \infty)$ be a semimartingale with $Z(0)=0$ a.s.

(i) If $\alpha>0, Z \in \mathcal{S}(\alpha)$, and $T$ is a stopping time, then $Z^{T} \in \mathcal{S}(\alpha)$ and $Z^{T-} \in$ $\mathcal{S}(\alpha)$

(ii) For every $\alpha>0$ there exist stopping times $0 \leqslant T_{0} \leqslant T_{1} \leqslant \cdots$ such that $\sup _{k} T_{k}=\infty$ a.s. and $Z^{T_{k}-} \in \mathcal{S}(\alpha)$ for all $k$.

It follows that for every $T>0, \alpha>0, t_{0}>0$, and $Z \in H^{\infty}\left[0, t_{0}\right]$ there exist stopping times $0 \leqslant T_{0} \leqslant T_{1} \leqslant \cdots$ with $\sup _{k} T_{k}=\infty$ a.s. and such that $Z^{T_{k}-} \in \mathcal{S}(\alpha)$ for all $k$.

The next lemma extends Lemma 6.2 to more general semimartingales.

Lemma 6.5. Let $1 \leqslant p<\infty$, let $t_{0}>0$, and let $J \in S^{p}\left[0, t_{0}\right]$. Let $F$ : $\mathbb{D}\left[0, t_{0}\right] \rightarrow \mathbb{D}\left[0, t_{0}\right]$ be functional Lipschitz as in Definition 6.1, $F(0)=0$, and $\sup _{t \in\left[0, t_{0}\right]}|K(t)| \leqslant k$ a.s. for some constant $k$. Let $g:\left[0, t_{0}\right] \rightarrow \mathbb{R}$ and $R>0$ be such that (6.1) is satisfied. Let $Z \in H^{\infty}\left[0, t_{0}\right]$ with $Z \in \mathcal{S}(1 / 2 \gamma)$, where

$$
\gamma=c_{p} k R,
$$

and let $T$ be a stopping time. Then

$$
X(t)=J^{T-}(t)+\left(\int_{0}^{\bullet} g(\bullet-s) F(X)(s-) \mathrm{d} Z(s)\right)^{T-}(t), \quad 0 \leqslant t \leqslant t_{0},
$$

has a unique solution $X \in S^{p}\left[0, t_{0}\right]$.

Proof. Let $S_{0}, S_{1}, \ldots, S_{\ell}$ be stopping times such that $0=S_{0} \leqslant S_{1} \leqslant \cdots \leqslant S_{\ell}$, $Z=Z^{S_{\ell-}}$, and $\left\|\left(Z-Z^{S_{i}}\right)^{S_{i+1}-}\right\|_{H^{\infty}} \leqslant 1 / 2 \gamma$ for $0 \leqslant i \leqslant \ell-1$ (these exist because $Z \in \mathcal{S}(1 / 2 \gamma))$. Let $T_{i}:=S_{i} \wedge T, i=0, \ldots, \ell$. Then $0=T_{0} \leqslant T_{1} \leqslant \cdots \leqslant T_{\ell}$ and $\left.\left\|\left(Z-Z^{T_{i}}\right)^{T_{i+1}-}\right\|_{H^{\infty}}=\|\left(Z-Z^{S_{i}}\right)^{S_{i+1}-}\right)^{T-} \|_{H^{\infty}} \leqslant 1 / 2 \gamma$. We argue by induction on $i$.

Suppose that $i \in\{0, \ldots, \ell-1\}$ is such that

$$
X(t)=J^{T_{i}-}(t)+\left(\int_{0}^{\bullet} g(\bullet-s) F(X)(s-) \mathrm{d} Z^{T_{i}-}(s)\right)^{T_{i}-}(t), \quad 0 \leqslant t \leqslant t_{0},
$$

has a unique solution $X \in S^{p}$. In order to simplify notations, we extend $F(U)(t):=$ $F(U)\left(t_{0}\right), J(t):=J\left(t_{0}\right)$, and $Z(t):=Z\left(t_{0}\right)$ for $t \geqslant t_{0}$ and $U \in \mathbb{D}\left[0, t_{0}\right]$. Further we interpret $[c, b]=\emptyset$ if $c>b$. Let

$$
Y:=X+\left(\Delta J\left(T_{i}\right)+\Delta\left(\int_{0}^{\bullet} g(\bullet-s) F(X)(s-) \mathrm{d} Z(s)\right)\left(T_{i}\right)\right) 1_{\left[T_{i}, t_{0}\right]} .
$$

Observe that $Y^{T_{i}-}=X^{T_{i}-}$ and $Y^{T_{i}}=Y$.

CLAIM: $Y$ is the unique solution in $S^{p}$ of

$$
V(t)=J^{T_{i}}(t)+\left(\int_{0}^{\bullet} g(\bullet-s) F(V)(s-) \mathrm{d} Z^{T_{i}}(s)\right)^{T_{i}}(t), \quad 0 \leqslant t \leqslant t_{0} .
$$


Proof of the claim: We have $Y^{T_{i}-}=X^{T_{i}-}$, so $F(Y)^{T_{i}-}=F(X)^{T_{i}-}$ and

$$
\begin{aligned}
J^{T_{i}}(t) & +\left(\int_{0}^{\bullet} g(\bullet-s) F(Y)(s-) \mathrm{d} Z(s)\right)^{T_{i}}(t) \\
= & J^{T_{i}}(t)+\left(\int_{0}^{\bullet} g(\bullet-s) F(X)(s-) \mathrm{d} Z(s)\right)^{T_{i}}(t) \\
= & X(t)+\Delta J\left(T_{i}\right) 1_{\left[T_{i}, t_{0}\right]}(t) \\
& \quad+\Delta\left(\int_{0}^{\bullet} g(\bullet-s) F(X)(s-) \mathrm{d} Z(s)\right)\left(T_{i}\right) 1_{\left[T_{i}, t_{0}\right]}(t) \\
= & Y(t), \quad 0 \leqslant t \leqslant t_{0} .
\end{aligned}
$$

Further,

$$
\left\|\sup _{s \in\left[0, t_{0}\right]}|Y(s)|\right\|_{L^{p}} \leqslant\|X\|_{S^{p}}+\|J\|_{S^{p}}+2 R\|F(X)\|_{S^{p}}\|Z\|_{H^{\infty}},
$$

so $Y \in S^{p}$. To see uniqueness, suppose that $V \in S^{p}$ is another solution of (6.2). Then $V^{T_{i}-}$ satisfies the equation for $X$, so $V^{T_{i}-}=X^{T_{i}-}=Y^{T_{i}-}$. From (6.2) it is clear that $V=V^{T_{i}}$ and that

$$
\begin{aligned}
V^{T_{i}}-V^{T_{i}-} & =\left(\Delta J\left(T_{i}\right)+\Delta\left(\int_{0}^{\bullet} g(\bullet-s) F(X)(s-) \mathrm{d} Z(s)\right)\left(T_{i}\right)\right) 1_{\left[T_{i}, t_{0}\right]} \\
& =Y^{T_{i}}-Y^{T_{i}-}
\end{aligned}
$$

so that $V=Y$.

Next, denote $D_{i} U:=\left(U-U^{T_{i}}\right)^{T_{i+1}-}, U \in \mathbb{D}\left[0, t_{0}\right]$. Consider

$$
\begin{aligned}
U(t)=( & D_{i} J(t)+\left(\int_{0}^{\bullet} g(\bullet-s) F(Y)(s-) \mathrm{d} Z^{T_{i+1}-}(s)\right)^{T_{i+1}-}(t) \\
& \left.-\left(\int_{0}^{\bullet} g(\bullet-s) F(Y)(s-) \mathrm{d} Z(s)\right)^{T_{i} \wedge T_{i+1}-}(t)\right) \\
& +\left(\int_{0}^{\bullet} g(\bullet-s) G(U)(s-) \mathrm{d} D_{i} Z(s)\right)^{T_{i+1}-}(t), \quad 0 \leqslant t \leqslant t_{0},
\end{aligned}
$$

where

$$
G(U)=F(Y+U)-F(Y), \quad U \in \mathbb{D}\left[0, t_{0}\right] .
$$

CLAIM: (a) equation (6.3) has a unique solution $U$ in $S^{p}$, and (b) the process $V:=U+Y^{T_{i+1}-}$ is the unique solution of

$$
V(t)=J^{T_{i+1}-}(t)+\left(\int_{0}^{\bullet} g(\bullet-s) F(V)(s-) \mathrm{d} Z^{T_{i+1}-}(s)\right)^{T_{i+1}-}(t), \quad 0 \leqslant t \leqslant t_{0} .
$$

Proof of the claim: (a) Observe that the sum of the first three terms of (6.3) is a member of $S^{p}, G$ is functional Lipschitz with $G(0)=0$ and satisfying the same estimates as $F$, and

$$
\left\|D_{i} Z(s)\right\|_{H^{\infty}}=\left\|\left(Z-Z^{T_{i}}\right)^{T_{i+1}-}\right\|_{H^{\infty}}<1 / 2 \gamma .
$$

Now apply Lemma 6.2. 
(b) From (6.3) it is clear that $U=U^{T_{i+1}-}$ and $U^{T_{i}-}=0$ Consequently, $F(Y+$ $U)^{T_{i}-}=F(Y)^{T_{i}-}$. For $0 \leqslant t \leqslant t_{0}$ we have, due to $(6.2)$,

$$
\begin{aligned}
U(t)+ & Y^{T_{i+1}-}(t) \\
= & J^{T_{i+1}-}(t)-\left(J^{T_{i}}\right)^{T_{i+1}-}(t)+\left(\int_{0}^{\bullet} g(\bullet-s) F(Y)(s-) \mathrm{d} Z^{T_{i+1}-}(s)\right)^{T_{i+1}-}(t) \\
& -\left(\int_{0}^{\bullet} g(\bullet-s) F(Y)(s-) \mathrm{d} Z(s)\right)^{T_{i} \wedge T_{i+1}-}(t) \\
& +\left(\int_{0}^{\bullet} g(\bullet-s) F(Y+U)(s-) \mathrm{d} Z^{T_{i+1}-}(s)\right)^{T_{i+1}-}(t) \\
& -\left(\int_{0}^{\bullet} g(\bullet-s) F(Y+U)(s-) \mathrm{d}\left(Z^{T_{i}}\right)^{T_{i+1}-}(s)\right)^{T_{i+1}-}(t) \\
& -\left(\int_{0}^{\bullet} g(\bullet-s) F(Y)(s-) \mathrm{d} D_{i} Z(s)\right)^{T_{i+1}-}(t)+Y^{T_{i+1}-}(t) \\
= & J^{T_{i+1}-}(t)+\left(\int_{0}^{\bullet} g(\bullet-s) F(Y+U)(s-) \mathrm{d} Z^{T_{i+1}-}(s)\right)^{T_{i+1}-}(t) .
\end{aligned}
$$

For any solution $V$, the process $V-Y^{T_{i+1}-}$ satisfies (6.3) and therefore equals $U$.

Conclusion: if

$$
X(t)=J^{T_{i}-}(t)+\left(\int_{0}^{\bullet} g(\bullet-s) F(X)(s-) \mathrm{d} Z^{T_{i}-}(s)\right)^{T_{i}-}(t), \quad 0 \leqslant t \leqslant t_{0},
$$

has a unique solution $X$ in $S^{p}$, then the equation

$$
X(t)=J^{T_{i+1}-}(t)+\left(\int_{0}^{\bullet} g(\bullet-s) F(X)(s-) \mathrm{d} Z^{T_{i+1}-}(s)\right)^{T_{i+1}-}(t), \quad 0 \leqslant t \leqslant t_{0},
$$

has a unique solution $X$ in $S^{p}$. As for $i=0, X=0$ is the unique solution $X$ in $S^{p}$, we find that there exists a unique $V \in S^{p}$ such that

$$
V(t)=J^{T_{\ell}-}(t)-\left(\int_{0}^{\bullet} g(\bullet-s) F(V)(s-) \mathrm{d} Z^{T_{\ell}-}(s)\right)^{T_{\ell}-}(t), \quad 0 \leqslant t \leqslant t_{0} .
$$

Finally, let

$$
X(t):=J^{T-}(t)+\left(\int_{0}^{\bullet} g(\bullet-s) F(V)(s-) \mathrm{d} Z(s)\right)^{T-}(t), \quad t \in\left[0, t_{0}\right] .
$$

Because $T_{\ell}=T \wedge S_{\ell}$ and $Z^{S_{\ell-}}=Z$ we have $X^{T_{\ell}-}=V$ and hence

$$
\begin{gathered}
\left(\int_{0}^{\bullet} g(\bullet-s) F(X)(s-) \mathrm{d} Z(s)\right)^{T-}(t)=\left(\int_{0}^{\bullet} g(\bullet-s) F\left(X^{T_{\ell}-}\right)(s-) \mathrm{d} Z^{T_{\ell}-}(s)\right)^{T-} \\
=\left(\int_{0}^{\bullet} g(\bullet-s) F(V)(s-) \mathrm{d} Z(s)\right)^{T-}=X(t)-J^{T-}(t), t \in\left[0, t_{0}\right] .
\end{gathered}
$$

We will increase the generality of the assumptions building on Lemma 6.5 in Lemma 6.8 below. The next two lemmas are needed in the proof of Lemma 6.8 .

Lemma 6.6. Let $X \in \mathbb{D}[0, \infty)$ and let $1 \leqslant p \leqslant \infty$. Then there exist stopping times $0=T_{0} \leqslant T_{1} \leqslant \cdots$ such that $\sup _{n} T_{n}=\infty$ a.s. and $X^{T_{n}-} \in S^{p}[0, \infty)$ for all $n$. 
Proof. Define $T_{0}:=0$,

$$
T_{n}:=\inf \{t \geqslant 0: X(t)>n\}, \quad n=1,2, \ldots
$$

(Here $\inf \emptyset:=\infty$.) Then for each $n \in \mathbb{N}, T_{n}$ is a stopping time, and

$$
\sup _{s \geqslant 0}\left|X^{T_{n}-}(s)\right| \leqslant n
$$

so that $X^{T_{n}-} \in S^{p}$. For a.e. $\omega$ and every $t \geqslant 0$, there is an $n \in \mathbb{N}$ such that

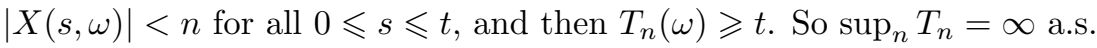

Lemma 6.7. Let $Y \in \mathbb{D}[0, \infty)$ and let $Z \in \mathbb{D}[0, \infty)$ be a semimartingale with $Z(0)=0$ a.s. Let $1 \leqslant p<\infty$ and let $g:[0, \infty) \rightarrow \mathbb{R}$ be a function such that for every $t_{0}>0$ there exists a constant $R>0$ such that (6.1) is satisfied. Then

$$
\left(\int_{0}^{t} g(t-s) Y(s-) \mathrm{d} Z(s)\right)_{t \geqslant 0}
$$

is a semimartingale.

Proof. Use Theorem 6.4 and Lemma 6.6 to choose stopping times $0=T_{0} \leqslant T_{1} \leqslant \cdots$ with $\sup _{k} T_{k}=\infty$ a.s. such that $Y^{T_{k}} \in S^{p}[0, \infty)$ and $Z^{T_{k}-} \in H^{\infty}[0, \infty)$ for each $k$. Then for each $t_{0}>0,\left(\int_{0}^{\bullet} g(\bullet-s) Y^{T_{k}-}(s-) \mathrm{d} Z^{T_{k}-}(s)\right)_{t \in\left[0, t_{0}\right]} \in H^{p}\left[0, t_{0}\right]$. Hence

$$
\left(\int_{0}^{\bullet} g(\bullet-s) Y(s-) \mathrm{d} Z(s)\right)^{T_{k} \wedge t_{0}-}=\left(\int_{0}^{\bullet} g(\bullet-s) Y^{T_{k}-}(s-) \mathrm{d} Z^{T_{k}-}(s)\right)^{T_{k} \wedge t_{0}-}
$$

equals a stopped semimartingale. It follows that $\int_{0}^{\bullet} g(\bullet-s) Y(s-) \mathrm{d} Z(s)$ is a local semimartingale and hence a semimartingale, by [5, Proposition I.4.25a) and b)].

Lemma 6.8. Let $Z$ be a semimartingale, $J \in \mathbb{D}[0, \infty)$, and let $F: \mathbb{D}[0, \infty) \rightarrow$ $\mathbb{D}[0, \infty)$ be functional Lipschitz. Let $g:[0, \infty) \rightarrow \mathbb{R}$ be a function such that for every $t_{0}>0$ there exists a constant $R>0$ such that (6.1) is satisfied. Then

$$
X(t)=J(t)+\int_{0}^{t} g(t-s) F(X)(s-) \mathrm{d} Z(s), \quad t \geqslant 0,
$$

has a unique solution $X$ in $\mathbb{D}[0, \infty)$. If $J$ is a semimartingale, then so is $X$.

Proof. We use the notations of Definition 6.1. As $F(X)\left(0_{-}\right)=0$ for all $X$, we may assume that $Z(0)=0$. We begin by replacing $J$ by $J+\int_{0}^{\bullet} g(\bullet-s) F(0)(s-) \mathrm{d} Z(s)$ and $F$ by $F(\bullet)-F(0)$. Thus we may assume that $F(0)=0$.

CLAIM: Let $t_{0}>0$. Suppose that $|K(t, \omega)| \leqslant k$ for a.e. $\omega$ and all $0 \leqslant t \leqslant t_{0}$. Let $S$ be a stopping time. Then there is a unique process $X \in \mathbb{D}$ such that

$$
X(t)=J^{S-}(t)+\left(\int_{0}^{\bullet} r(\bullet-s) F(X)(s-) \mathrm{d} Z(s)\right)^{S-}(t), \quad 0 \leqslant t \leqslant t_{0} .
$$

Proof of the claim: Let $R>0$ be a constant corresponding to $t_{0}$ such that (6.1) is satisfied. Let

$$
\gamma:=c_{p} k R \text {. }
$$

For every stopping time $T$ such that $J^{T-} \in S^{2}$ and $Z^{T-} \in \mathcal{S}(1 / 2 \gamma)$, Lemma 6.5 says that there is a unique $X_{T} \in S^{2}$ such that

$$
X_{T}=\left(J^{T-}\right)^{S-}+\left(\int_{0}^{\bullet} g(\bullet-s) F\left(X_{T}\right)(s-) \mathrm{d} Z^{T-}(s)\right)^{S-} .
$$


By uniqueness we have for any two such stopping times $T_{1}$ and $T_{2}$ that $X_{T_{1}}^{T_{3}-}=$ $X_{T_{2}}^{T_{3}-}$, where $T_{3}=T_{1} \wedge T_{2}$. According to Theorem 6.4 and Lemma 6.6, there exist stopping times $0=T_{0} \leqslant T_{1} \leqslant T_{2} \leqslant \cdots$ such that $\sup _{\ell} T_{\ell}=\infty$ a.s., $J^{T_{\ell}-} \in S^{2}$, and $Z^{T_{\ell}-} \in \mathcal{S}(1 / 2 \gamma)$ for all $\ell$. Define

$$
X(t):=\sum_{\ell=1}^{\infty} X_{T_{\ell}}(t) 1_{\left[T_{\ell-1}, T_{\ell}\right)}(t), \quad 0 \leqslant t \leqslant t_{0} .
$$

Then $(X(t))_{t \geqslant 0}$ is an adapted càdlàg process and $X^{S-}=X$. Further, for $\ell \geqslant 1$, $X^{T_{\ell}-}=X_{T_{\ell}}$, and

$$
\begin{aligned}
& \left(\left(J+\int_{0}^{\bullet} g(\bullet-s) F(X)(s-) \mathrm{d} Z(s)\right)^{S-}\right)^{T_{\ell}-} \\
& =\left(J^{T_{\ell}-}\right)^{S-}+\left(\left(\int_{0}^{\bullet} g(\bullet-s) F(X)^{T_{\ell}-}(s-) \mathrm{d} Z(s)\right)^{T_{\ell}-}\right)^{S-} \\
& =\left(J^{T_{\ell}-}\right)^{S-}+\left(\left(\int_{0}^{\bullet} g(\bullet-s) F\left(X_{T_{\ell}}\right)(s-) \mathrm{d} Z(s)\right)^{T_{\ell}-}\right)^{S-} \\
& =X_{T_{\ell}}=X^{T_{\ell}-} .
\end{aligned}
$$

It follows that

$$
X(t)=J^{S-}(t)+\left(\int_{0}^{\bullet} g(\bullet-s) F(X)(s-) \mathrm{d} Z(s)\right)^{S-}(t), \quad 0 \leqslant t \leqslant t_{0} .
$$

To show uniqueness, let $Y$ also be an adapted càdlàg process such that

$$
Y(t)=J^{S-}(t)+\left(\int_{0}^{\bullet} g(\bullet-s) F(Y)(s-) \mathrm{d} Z(s)\right)^{S-}(t), \quad 0 \leqslant t \leqslant t_{0} .
$$

According to Lemma 6.6 there exist stopping times $0=S_{0} \leqslant S_{1} \leqslant \cdots$ with $\sup _{\ell} S_{\ell}=\infty$ a.s. and $Y^{S_{\ell}-} \in S^{2}$ for all $\ell$. Then $Y^{\left(S_{\ell} \wedge T_{\ell}\right)-}$ satisfies the same equation as $X_{T_{\ell}}^{S_{\ell}}$ and by uniqueness we obtain

$$
Y^{\left(S_{\ell} \wedge T_{\ell}\right)-}=X_{T_{\ell}}^{S_{\ell}-}=X^{\left(S_{\ell} \wedge T_{\ell}\right)-} .
$$

Since $\sup _{\ell} S_{\ell} \wedge T_{\ell}=\infty$ a.s., it follows that $X=Y$.

Next, fix $t_{0}>0$. For $n=1,2,3, \ldots$ define the stopping time

$$
T_{n}(\omega):=\inf \left\{t \in\left[0, t_{0}\right]: K(t, \omega)>n\right\}, \quad \omega \in \Omega,
$$

where $\inf \emptyset:=\infty$. Then $0=T_{0} \leqslant T_{1} \leqslant \cdots$ a.s. and $\sup _{n} T_{n}=\infty$ a.s. Define

$$
F_{n}(X)(t):=F(X)^{T_{n}-}(t), \quad 0 \leqslant t \leqslant t_{0}, X \in \mathbb{D}\left[0, t_{0}\right], n=1,2, \ldots
$$

Then $F_{n}: \mathbb{D}\left[0, t_{0}\right] \rightarrow \mathbb{D}\left[0, t_{0}\right]$ is functional Lipschitz, $\left|F_{n}(X)(t)-F_{n}(Y)(t)\right| \leqslant$ $n \sup _{0 \leqslant s \leqslant t}|X(s)-Y(s)|$, for all $X, Y \in \mathbb{D}\left[0, t_{0}\right]$, and $F_{n}(0)=0$. By the first claim, there exists therefore for each $n$ a unique $Y_{n} \in \mathbb{D}\left[0, t_{0}\right]$ such that

$$
Y_{n}(t)=J^{T_{n}-}(t)+\left(\int_{0}^{\bullet} g(\bullet-s) F_{n}\left(Y_{n}\right)(s-) \mathrm{d} Z(s)\right)^{T_{n}-}(t), \quad 0 \leqslant t \leqslant t_{n} .
$$

Then by uniqueness, $Y_{m}^{T_{n}-}=Y_{n}$ for every $m \geqslant n$. Define

$$
Y(t):=\sum_{n=1}^{\infty} Y_{n}(t) 1_{\left[T_{n-1}, T_{n}\right)}(t), \quad 0 \leqslant t \leqslant t_{0}
$$


Then $Y \in \mathbb{D}\left[0, t_{0}\right]$ and $Y^{T_{n}-}=Y_{n}$ for $n=1,2, \ldots$ Further, for each $n$,

$$
\begin{aligned}
Y^{T_{n}-} & =J^{T_{n}-}+\left(\int_{0}^{\bullet} g(\bullet-s) F_{n}\left(Y_{n}\right)(s-) \mathrm{d} Z(s)\right)^{T_{n}-} \\
& =J^{T_{n}-}+\left(\int_{0}^{\bullet} g(\bullet-s) F(Y)(s-) \mathrm{d} Z(s)\right)^{T_{n}-} .
\end{aligned}
$$

Hence $Y$ satisfies

$$
Y(t)=J(t)+\int_{0}^{t} g(t-s) F(Y)(s-) \mathrm{d} Z(s), \quad 0 \leqslant t \leqslant t_{0} .
$$

Moreover, $Y$ is the unique solution of the latter equation. Indeed, if $V$ is a solution as well, then $V^{T_{n}-}$ satisfies the defining equation for $Y_{n}$ and therefore $V^{T_{n}-}=Y_{n}=$ $Y^{T_{n}-}$ for all $n$. This implies $V=Y$.

Finally, we can vary $t_{0}$ and glue solutions together to obtain a unique $Y \in \mathbb{D}$ such that

$$
Y(t)=J(t)+\int_{0}^{t} g(t-s) F(Y)(s-) \mathrm{d} Z(s), t \geqslant 0 .
$$

It follows from Lemma 6.7 that $X$ is a semimartingale whenever $J$ is a semimartingale.

Theorem 1.3 follows from Lemma 5.3 and Lemma 6.8. Notice that the function $g$ in Theorem 1.3 need not be continuous. In this way Theorem 1.3 generalizes [8].

\section{Variation-of-constants formula for SDDE with linear drift}

It is the aim of this section to prove the next variation-of-constants formula.

Theorem 7.1. Let $\mu$ be a finite signed Borel measure on $(-\infty, 0]$ and let $g: \mathbb{R} \rightarrow \mathbb{R}$ be the unique solution of

$$
\begin{cases}\left.g\right|_{[0, \infty)} ^{\prime}=\int_{(-\infty, 0]} g(\bullet+a) \mu(\mathrm{d} a) & \text { Lebesgue-a.e. on }[0, \infty), \\ g(0)=1 & t<0,\end{cases}
$$

with $\left.g\right|_{[0, \infty)}$ absolutely continuous. Let $F: \mathbb{D}[0, \infty) \rightarrow \mathbb{D}[0, \infty)$ be functional Lipschitz and assume that $F(X)$ is bounded for every $X \in \mathbb{D}[0, \infty)$. Let $J$ and $Z$ be semimartingales. Then a process $X \in \mathbb{D}[0, \infty)$ satisfies

$$
\begin{aligned}
X(t)=J(t) & +\int_{0}^{t} \int_{(-s, 0]} X(s+a) \mu(\mathrm{d} a) d s \\
& +\int_{0}^{t} F(X)(s-) \mathrm{d} Z(s), \quad t \geqslant 0 .
\end{aligned}
$$

if and only if it satisfies the variation-of-constants formula

$$
\begin{aligned}
X(t)=g(t) & J(0)+\int_{0}^{t} g(t-s) \mathrm{d}(J(s)-J(0)) \\
& +\int_{0}^{t} g(t-s) F(X)(s-) \mathrm{d} Z(s), \quad t \geqslant 0 .
\end{aligned}
$$

Moreover, there exists one and only one $X \in \mathbb{D}[0, \infty)$ satisfying (7.2) and (7.3). 
It is well known that (7.1) has a unique solution $g: \mathbb{R} \rightarrow \mathbb{R}$ with $\left.g\right|_{[0, \infty)}$ absolutely continuous (see [4]). In particular, $\left.g\right|_{[0, T]}$ is of bounded variation for any $T>0$. It is straightforward to show that therefore $\int_{(-\infty, 0]} g(\bullet+a) \mu(\mathrm{d} a)$ is of bounded variation on $[0, T]$ and hence $\left.g\right|_{[0, T]} \in B V^{1}[0, T]$ for every $T>0$.

The proof of Theorem 7.1 is as follows. Due to Theorem 1.3, there exists a solution of (7.3). By means of a stochastic Fubini argument, we will show that this solution also satisfies (7.2). As equation (7.2) has only one solution, we then know that the solutions of (7.2) and (7.3) coincide and the proof is complete. The Fubini argument is given next.

Lemma 7.2. Let $\mu$ be a finite signed Borel measure on $(-\infty, 0]$ and let $g: \mathbb{R} \rightarrow \mathbb{R}$ be the solution of (7.1) with $\left.g\right|_{[0, \infty)}$ absolutely continuous. Let $F: \mathbb{D}[0, \infty) \rightarrow \mathbb{D}[0, \infty)$ be functional Lipschitz and assume that $F(X)$ is bounded for every $X \in \mathbb{D}[0, \infty)$. Let $(Z(t))_{t \geqslant 0}$ and $(J(t))_{t \geqslant 0}$ be semimartingales with $J(0)=0$ a.s. If $X \in \mathbb{D}[0, \infty)$ satisfies

$$
\begin{aligned}
X(t)=g(t) X(0)+\int_{0}^{t} g(t-s) \mathrm{d} J(s) & \\
& +\int_{0}^{t} g(t-s) F(X)(s-) \mathrm{d} Z(s), \quad t \geqslant 0,
\end{aligned}
$$

then

$$
\begin{aligned}
X(t)=X(0) & +J(t)+\int_{0}^{t} \int_{(-s, 0]} X(s+a) \mu(\mathrm{d} a) \mathrm{d} s \\
& +\int_{0}^{t} F(X)(s-) \mathrm{d} Z(s), \quad t \geqslant 0,
\end{aligned}
$$

Proof. We will first apply a stochastic Fubini theorem twice to prove the identity

$$
\begin{aligned}
\int_{0}^{T} & \int_{(-\infty, 0]} \int_{0}^{(s+a)^{+}} g(s+a-m) F(X)(m-) \mathrm{d} Z(m) \mu(\mathrm{d} a) \mathrm{d} s \\
& =\int_{0}^{T} g(T-m) F(X)(m-) \mathrm{d} Z(m)-\int_{0}^{T} F(X)(m-) \mathrm{d} Z(m),
\end{aligned}
$$

for any $T \geqslant 0$ and any $X \in \mathbb{D}$. Since we will only evaluate $g$ on $(-\infty, T]$, we may assume that $g$ is bounded. Fix an $s \in[0, T]$. The map $(a, t, \omega) \mapsto g(s+a-$ t) $F(X)(t-)(\omega)$ is bounded and $\mathcal{B}((-\infty, 0]) \otimes \mathcal{P}$-measurable. Here $\mathcal{P}$ denotes the predictable $\sigma$-algebra, which is the smallest $\sigma$-algebra in $[0, \infty) \times \Omega$ such that each adapted process with almost surely càglàd (that is, left continuous with existence of right limits) paths is measurable (see [9, p.25]). Then [9, Theorem IV.44, p.158] asserts that for each $a \in(-\infty, 0]$ we can choose an adapted càdlàg version of the stochastic integral

$$
\left(\int_{0}^{t} g(s+a-m) F(X)(m-) \mathrm{d} Z(m)\right)_{t \geqslant 0}
$$

such that we obtain a $\mathcal{B}((-\infty, 0]) \otimes \mathcal{B}([0, \infty)) \otimes \mathcal{F}$-measurable map in the variables $(a, t, \omega)$. With this choice of versions, [9, Theorem IV.45, p.159] yields that

$$
\begin{aligned}
\int_{(-\infty, 0]} & \left(\int_{0}^{t} g(s+a-m) F(X)(m-) \mathrm{d} Z(m)\right) \mu(\mathrm{d} a) \\
= & \int_{0}^{t}\left(\int_{(-\infty, 0]} g(s+a-m) F(X)(m-) \mu(\mathrm{d} a)\right) \mathrm{d} Z(m) \text { a.s. }
\end{aligned}
$$


for every $t \geqslant 0$. In particular, since $g(\theta)=0$ for $\theta<0$ we obtain for $t \geqslant s$,

$$
\begin{aligned}
\int_{(-\infty, 0]} & \left(\int_{0}^{(s+a)^{+}} g(s+a-m) F(X)(m-) \mathrm{d} Z(m)\right) \mu(\mathrm{d} a) \\
= & \int_{0}^{t}\left(\int_{(-\infty, 0]} g(s+a-m) \mu(\mathrm{d} a) F(X)(m-)\right) \mathrm{d} Z(m) \text { a.s.. }
\end{aligned}
$$

The map $(s, m, \omega) \mapsto \int_{(-\infty, 0]} g(s+a-m) \mu(\mathrm{d} a) F(X)(m-)$ is bounded and measurable with respect to $\mathcal{B}([0, T]) \otimes \mathcal{P}$, because $(s, m) \mapsto \int_{(-\infty, 0]} g(s+a-m) \mu(\mathrm{d} a)$ is $\mathcal{B}([0, T]) \otimes \mathcal{P}$-measurable. Hence, again by $[9$, Theorem IV.44-45, p.158-9], we can choose for every $s \in[0, T]$ an adapted càdlàg version of

$$
\left(\int_{0}^{t} \int_{(-\infty, 0]} g(s+a-m) \mu(\mathrm{d} a) F(X)(m-) \mathrm{d} Z(m)\right)_{t \geqslant 0}
$$

such that we obtain an $\mathcal{B}([0, T]) \otimes \mathcal{B}([0, \infty)) \otimes \mathcal{F}$-measurable map, and with this choice of version we have

$$
\begin{aligned}
\int_{0}^{T} & \left(\int_{0}^{t} \int_{(-\infty, 0]} g(s+a-m) \mu(\mathrm{d} a) F(X)(m-) \mathrm{d} Z(m)\right) \mathrm{d} s \\
& =\int_{0}^{t}\left(\int_{0}^{T} \int_{(-\infty, 0]} g(s+a-m) \mu(\mathrm{d} a) F(X)(m-) \mathrm{d} s\right) \mathrm{d} Z(m) \text { a.s. }
\end{aligned}
$$

for every $t \geqslant 0$. Hence, as $g(\theta)=0$ for $\theta<0$, we obtain with $t=T$ that

$$
\begin{aligned}
\int_{0}^{T} & \left(\int_{0}^{T} \int_{(-\infty, 0]} g(s+a-m) \mu(\mathrm{d} a) \mathrm{d} s F(X)(m-) \mathrm{d} Z(m)\right) \mathrm{d} s \\
& =\int_{0}^{T}\left(\int_{m}^{T} \int_{(-\infty, 0]} g(s+a-m) \mu(\mathrm{d} a) \mathrm{d} s F(X)(m-)\right) \mathrm{d} Z(m) .
\end{aligned}
$$

Finally, we rewrite the right hand side by observing that for $m \geqslant 0$,

$$
\begin{aligned}
\int_{m}^{T} \int_{(-\infty, 0]} g(s+a-m) \mu(\mathrm{d} a) \mathrm{d} s & =\int_{0}^{T-m} \int_{(-\infty, 0]} g(s+a) \mu(\mathrm{d} a) \mathrm{d} s \\
& =\int_{0}^{T-m} g^{\prime}(s) \mathrm{d} s \\
& =g(T-m)-1,
\end{aligned}
$$

and we arrive at the identity (7.5).

Similarly, we have for any $T \geqslant 0$ that

$$
\begin{aligned}
\int_{0}^{T} & \int_{(-\infty, 0]} \int_{0}^{(s+a)^{+}} g(s+a-m) \mathrm{d} J(m) \mu(\mathrm{d} a) \mathrm{d} s \\
& =\int_{0}^{T} g(T-m) \mathrm{d} J(m)-\int_{0}^{T} \mathrm{~d} J(m) \\
& =\int_{0}^{T} g(T-m) \mathrm{d} J(m)-J(T) .
\end{aligned}
$$


Next assume that $X \in \mathbb{D}$ satisfies (7.4). Set $X(t):=0$ for $t<0$. Then

$$
\begin{aligned}
X(u)=g(u) X(0)+\int_{0}^{u^{+}} g(u-s) \mathrm{d} J(s) & \\
& +\int_{0}^{u^{+}} g(u-s) F(X)(s-) \mathrm{d} Z(s), \quad \text { for all } u \in \mathbb{R} .
\end{aligned}
$$

Therefore,

$$
\begin{aligned}
\int_{0}^{t} \int_{(-s, 0]} X(s+a) \mu(\mathrm{d} a) \mathrm{d} s=\int_{0}^{t} \int_{(-\infty, 0]} X(s+a) \mu(\mathrm{d} a) \mathrm{d} s \\
=\int_{0}^{t} \int_{(-\infty, 0]} g(s+a) \mu(\mathrm{d} a) \mathrm{d} s X(0) \\
\quad+\int_{0}^{t} \int_{(-\infty, 0]} \int_{0}^{(s+a)^{+}} g(s+a-m) \mathrm{d} J(m) \mu(\mathrm{d} a) \mathrm{d} s \\
\quad+\int_{0}^{t} \int_{(-\infty, 0]} \int_{0}^{(s+a)^{+}} g(s+a-m) F(X)(m-) \mathrm{d} Z(m) \mu(\mathrm{d} a) \mathrm{d} s \\
=\int_{0}^{t} g^{\prime}(s) \mathrm{d} s X(0)+\int_{0}^{t} g(t-m) \mathrm{d} J(m)-J(t) \\
\quad+\int_{0}^{t} g(t-m) F(X)(m-) \mathrm{d} Z(m) \\
\quad-\int_{0}^{t} F(X)(m-) \mathrm{d} Z(m) \\
=(g(t)-1) X(0)+\int_{0}^{t} g(t-m) \mathrm{d} J(m)-J(t) \\
\quad+\int_{0}^{t} g(t-m) F(X)(m-) \mathrm{d} Z(m) \\
\quad-\int_{0}^{t} F(X)(m-) \mathrm{d} Z(m) \\
=X(t)-X(0)-J(t)-\int_{0}^{t} F(X)(m-) \mathrm{d} Z(m),
\end{aligned}
$$

for all $t \geqslant 0$. Here the third equality is justified by the identities (7.5) and (7.6). Conclusion, $X$ satisfies

$$
\begin{aligned}
X(t)= & X(0)+J(t)+\int_{0}^{t} \int_{(-s, 0]} X(s+a) \mu(\mathrm{d} a) \mathrm{d} s \\
& +\int_{0}^{t} F(X)(m-) \mathrm{d} Z(m), \quad t \geqslant 0 .
\end{aligned}
$$

Acknowledgement. O. van Gaans acknowledges the financial support provided through the European Community's Human Potential Programme under contracts HPRN-CT-2000-00100, DYNSTOCH and HPRN-CT-2002-00281.

\section{References}

[1] G. Da Prato and J. Zabczyk, Stochastic Equations in Infinite Dimensions, Encyclopedia of mathematics and its applications, Vol. 45, Cambridge University Press, Cambridge, 1992. 
[2] Claude Dellacherie and Paul-André Meyer, Probabilité et potentiel, Chapitre $V$ à VIII, Théorie des martingales, Édition entièrement refondue, Hermann, Paris, 1980.

[3] M. Émery, Stabilité des solutions des équations différentielles stochastiques applications aux integrales multiplicatives stochastiques, Z. Wahrscheinlichkeitstheorie verw. Gebiete 41 (1978), 241-262.

[4] Jack K. Hale and Sjoerd M. Verduyn Lunel, Introduction to functional differential equations, Applied Mathematical Sciences 99, Springer-Verlag, New York, 1993.

[5] Jean Jacod and Albert. N. Shiryaev, Limit theorems for stochastic processes, Second edition, Springer-Verlag, Berlin, Heidelberg, 2003.

[6] Olav Kallenberg, Foundations of Modern Probability, Second Edition, SpringerVerlag, New York, 2002.

[7] Ioannis Karatzas and Steven E. Shreve, Brownian Motion and Stochastic Calculus, Second Ed., Graduate Texts in Mathematics 113, Springer-Verlag, New York, 1991.

[8] Philip Protter, Volterra equations driven by semimartingales, Ann. Probab. 13 (1985) no. 2, 519-530.

[9] Philip Protter, Stochastic Integration and Differential Equations, A New Approach, Springer-Verlag, Berlin, Heidelberg, 1990.

[10] M. Reiss, M. Riedle, and O. van Gaans, Delay differential equations driven by Lévy processes: stationarity and Feller properties, in preparation. 